\title{
Poor-data and data-poor species stock assessment using a Bayesian hierarchical approach
}

\author{
Yan Jiao, ${ }^{1,4}$ Enric Cortés, ${ }^{2}$ Kate Andrews, ${ }^{2}$ and Feng Guo ${ }^{3}$ \\ ${ }^{1}$ Department of Fisheries and Wildlife Sciences, Virginia Polytechnic Institute and State University, \\ Blacksburg, Virginia 24061-0321 USA \\ ${ }^{2}$ National Marine Fisheries Service, Panama City Laboratory, Panama City, Florida 32408 USA \\ ${ }^{3}$ Department of Statistics, Virginia Polytechnic Institute and State University, Blacksburg, Virginia 24061-0439 USA
}

\begin{abstract}
Appropriate inference for stocks or species with low-quality data (poor data) or limited data (data poor) is extremely important. Hierarchical Bayesian methods are especially applicable to small-area, small-sample-size estimation problems because they allow poor-data species to borrow strength from species with good-quality data. We used a hammerhead shark complex as an example to investigate the advantages of using hierarchical Bayesian models in assessing the status of poor-data and data-poor exploited species. The hammerhead shark complex (Sphyrna spp.) along the Atlantic and Gulf of Mexico coasts of the United States is composed of three species: the scalloped hammerhead (S. lewini), the great hammerhead $(S$. mokarran), and the smooth hammerhead (S. zygaena) sharks. The scalloped hammerhead comprises $70-80 \%$ of the catch and has catch and relative abundance data of good quality, whereas great and smooth hammerheads have relative abundance indices that are both limited and of low quality presumably because of low stock density and limited sampling. Four hierarchical Bayesian state-space surplus production models were developed to simulate variability in population growth rates, carrying capacity, and catchability of the species. The results from the hierarchical Bayesian models were considerably more robust than those of the nonhierarchical models. The hierarchical Bayesian approach represents an intermediate strategy between traditional models that assume different population parameters for each species and those that assume all species share identical parameters. Use of the hierarchical Bayesian approach is suggested for future hammerhead shark stock assessments and for modeling fish complexes with species-specific data, because the poor-data species can borrow strength from the species with good data, making the estimation more stable and robust.
\end{abstract}

Key words: Bayesian hierarchical model; data-poor assessment; hammerhead shark; fish complex; population dynamics; small sample size.

\section{INTRODUCTION}

It is widely recognized by analysts that population assessments of species that have poor data (where quality is poor) or are data poor (where data are limited in quantity) are very challenging. Because of this dual limitation, assessments of the status of exploited populations are often highly uncertain. Assessments of poor-data and data-poor species are thus often based on life history information (e.g., matrix models and perrecruit analysis; Mace and Sissenwine 1993, Fujiwara and Caswell 2001, Cortés 2002a) or abundance indices or catch histories (e.g., an index method; NEFSC 2008a, MacCall 2009, Northeast Data Poor Stocks Working Group 2009). Methods that can generally improve the reliability of stock assessments in poor-data and datapoor situations are thus urgently needed.

Manuscript received 12 March 2010; revised 7 February 2011; accepted 1 March 2011. Corresponding Editor: J. J. Barber.

${ }^{4}$ E-mail: yjiao@vt.edu
Hierarchical Bayesian methods are especially applicable to small-area and small-sample-size estimation problems because they allow poor-data species to borrow strength from species with good-quality data (Berger 1985, He and Sun 1998, 2000, Su et al. 2001, Gelman et al. 2004). Many hierarchical modeling analyses based on empirical data and simulations reveal the need for multilevel analysis of multilevel data (He and Sun 2000, Osborne 2000, Su et al. 2001). Hierarchical and multilevel models are the same in concept but different terminology is used according to the discipline. Additionally, hierarchical modeling analyses provide other benefits, such as easy modeling of cross-level interactions. This often allows for more interesting questions to be asked of the data, such as estimating parameters or variables of interest across experiments, locations, and times (Osborne 2000). With nested and hierarchical data being common in the social and other sciences, recent developments in Bayesian approaches, and the availability of high-performance computers that make hierarchical models solvable and 
accessible, it is important for fisheries researchers to become more acquainted with the application of Bayesian hierarchical techniques to assess the status of data-poor and poor-data species.

In this study, we used a hammerhead shark complex as an example to demonstrate the advantages of using hierarchical Bayesian models in assessing the status of poor-data and data-poor species. The hammerhead shark complex (Sphyrna spp.) along the Atlantic and Gulf of Mexico coasts of the USA is composed of three species: the scalloped hammerhead $(S$. lewini $)$, the great hammerhead (S. mokarran), and the smooth hammerhead (S. zygaena) sharks. Stock assessments of sharks often have had to focus on complexes, such as the large and small coastal sharks in the USA, because of the lack of species-specific data (Cortés 2002b, NMFS 2006). It is common to find that there are species with good data available and other species with only poor data in a fish complex (NMFS 2006, NEFSC 2008a, b). This is also true for other fisheries, such as those for the snappergrouper complex in the U.S. South Atlantic and Gulf of Mexico and some ground fishes in the U.S. North Atlantic (Polovina and Ralston 1987). The approach of hierarchical modeling for fish complexes used by Jiao et al. (2009a) was recently endorsed by the U.S. National Research Council and by a recent independent review for NOAA of the Hawaiian bottomfish fishery (NMFS 2009, NRC 2010). We further developed this study to deal with data-poor species in fish complexes.

In the examples used here, great and smooth hammerheads have relative abundance data that are both limited in quantity and of low quality, presumably because of low population densities and limited sampling (Hayes 2008). However, within the complex, the scalloped hammerhead shark comprises $70-80 \%$ of the catch and several series of relative abundance of fairly high quality are available, in contrast to the data for great and smooth hammerhead sharks. The three species are distributed in the same geographical areas and are exploited by the same fisheries. Because of the relatively low density of these sharks in the ocean and because of the limited efforts allocated to collecting data from both fishery-dependent and fishery-independent sources, the status of great and smooth hammerheads is largely unknown and the paucity of data led to inconclusive results (IUCN 2006, Hayes 2008). This situation, with one species having data of good quality and two species having limited, low-quality data, provides a good example to illustrate how hierarchical Bayesian models can improve assessment of the species with poor data by allowing them to borrow strength from the species with good-quality data (He and Sun 1998, Su et al. 2001, Gelman et al. 2004).

Population dynamics models that have been used for shark complexes are based on surplus production theory (see, e.g., NMFS 2006). In this study, variability among species is modeled with a hierarchical approach to address the problem of simulating population growth rates assigned to a particular species. In contrast to an overfitted model that would assign a different parameter value to each species, hierarchical models accommodate species or group differences but assume these differences are derived from an underlying distribution through hierarchical prior distributions (Wikle 2003, Gelman et al. 2004).

The hierarchical model was implemented in a Bayesian framework. Bayesian approaches commonly have been used in solving hierarchical models because of their flexibility in incorporating multiple levels of randomness and information from different sources. They are increasingly being used in fisheries stock assessment because of their ability to provide results for risk analyses of alternative management strategies and to incorporate prior knowledge of the fisheries into the assessment process (Berger 1985, McAllister and Kirkwood 1998, Gelman et al. 2004). Hierarchical Bayesian models have also shown robustness to prior specification and data uncertainty (Roberts and Rosenthal 2001).

Demographic data help us understand the basis of population dynamics and are especially useful when the populations are too sparse to assess (Kieth and Windberg 1978, Krebs et al. 2001). Population growth rate estimates based on demographic information can be used as prior knowledge in a hierarchical framework and help us to better simulate the population dynamics (Jiao et al. 2008, 2009a). In this example, demographic information of hammerhead sharks and other shark species is synthesized and used as prior information (Jiao et al. 2009a).

The goodness of fits of the Bayesian hierarchically structured surplus production models were compared with that of the traditional nonhierarchical surplus production model for each species using the deviance information criterion (DIC; Spiegelhalter et al. 2004). Robustness of the model fit was also used to compare models (Roberts and Rosenthal 2001). Our ultimate goal was to evaluate the Bayesian hierarchical model as an appropriate method to assess the population dynamics of data-poor and poor-data species. The framework developed here can be used to assess other species with similar data shortcomings.

\section{Methods}

\section{Data sources}

Data on hammerhead sharks were collected and summarized from several National Marine Fisheries Service (NMFS) sources (Beerkircher et al. 2002, Cortés et al. 2005, Cortés and Neer 2005, NMFS 2006, Hayes 2008; see Plate 1). Catch time series included recreational catches, commercial landings, and pelagic longline discards (Fig. 1). Relative abundance time series included the NMFS Mississippi bottomlongline shark survey (NMFS-LL-SE, in number of sharks per 10000 hook hours; Ingram et al. 2005), the NMFS Panama City gillnet shark survey (PCGN, in number of sharks per net per hour; Carlson and Bethea 

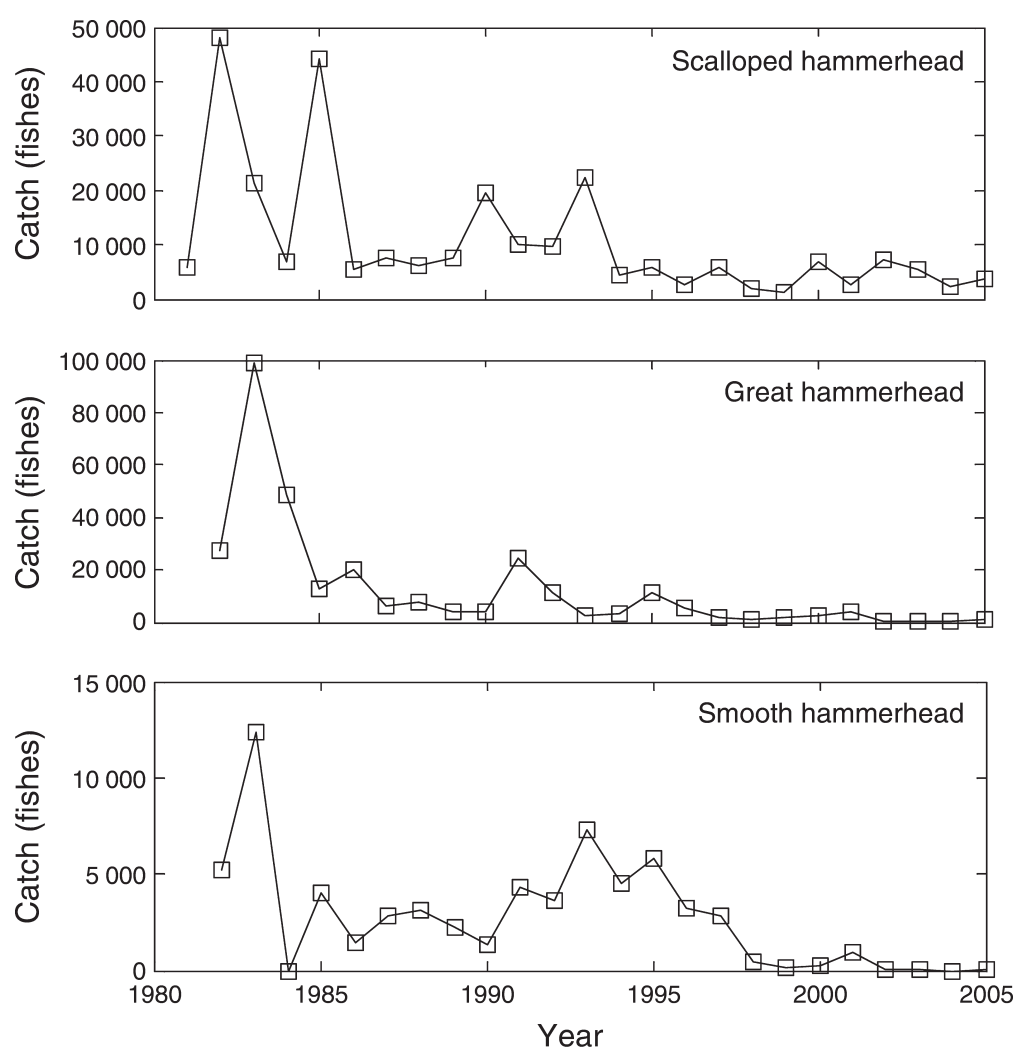

FIG. 1. Summarized catches (in numbers) of hammerhead sharks by species.

2005), the University of North Carolina longline survey (NCLL, in number of sharks per hook; Schwartz et al. 2007), the commercial shark fishery observer program (CSFOP, in number of sharks per 100 hooks per hour; Cortés et al. 2005), the shark drift gillnet observer program (GNOP in number of sharks per $10^{7} \mathrm{~m}^{2}$ per hour); Carlson et al. 2005), and the pelagic longline observer program (PLLOP, in number of sharks per 1000 hooks; Beerkircher et al. 2002; Fig. 2). Not all series have catch-per-unit-effort (CPUE) information for each species. Six series (NMFS-LL-SE, PCGN, NCLL, CSFOP, GNOP, and PLLOP) were available for scalloped hammerhead sharks because of the high portion of the hammerhead shark complex catch that this species represents, but only two relative abundance indices were available for great hammerhead (NMFSLL-SE and CSFOP) and one for smooth hammerhead sharks (PLLOP).

To compare the Bayesian hierarchically structured surplus production model to the traditional nonhierarchical surplus production model for each species, we first described the nonhierarchical surplus production model, followed by the hierarchical surplus production model with species-specific data. We named the nonhierarchical model M1. This model (M1) consisted of three models with the same structure, but was fitted to three different species, i.e., one model for each species. Four hierarchical models were developed in this study: the first model had population growth rate hierarchically structured (M2); the second one had population growth rate and carrying capacity hierarchically structured (M3); the third one set population growth rate and catchability to be hierarchically structured (M4); whereas the fifth one (M5) set population growth rate, carrying capacity and catchability all to be hierarchically structured.

\section{Bayesian state-space surplus production model for each species}

Because the data available on hammerhead sharks were not size, age, or stage structured and process error is often very important, we used a state-space surplus production model as the basic model structure (M1):

$$
\begin{aligned}
& E\left(N_{k, t}\right)=N_{k, t-1}+G_{k, t-1}-C_{k, t-1} \\
& E\left(I_{i, k, t}\right)=q_{i, k} N_{k, t}
\end{aligned}
$$

where $k$ indicates the species (here $k=1$ means scalloped hammerhead shark, $k=2$ means great hammerhead shark, and $k=3$ means smooth hammerhead shark); $i$ is the $i$ th type of relative abundance index (here $i$ ranges from 1 to 6 and represents relative abundance indices from surveys of NMFS-LL-SE, PCGN, NCLL, CSFOP, GNOP, and PLLOP, separately); $N_{k, t}$ is the abundance (in 1000 sharks) of species $k$ in year $t ; G_{k, t}$ is the production function of species $k$ in year $t ; C_{k, t}$ is the total catch (in 1000 sharks) of species $k$ in year $t$; and $q_{i, k}$ 

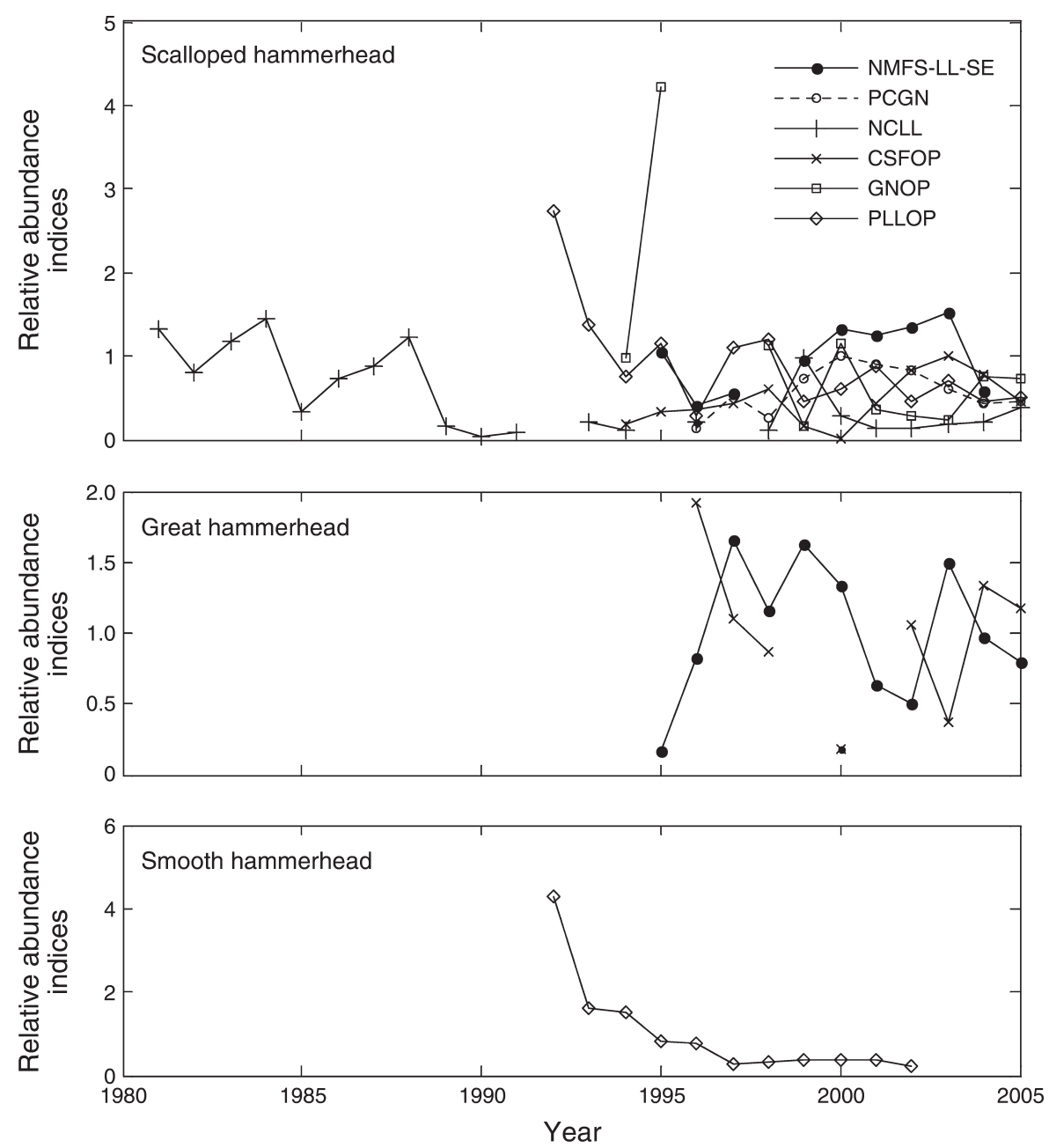

FIG. 2. Indices of relative abundance for hammerhead sharks by species. See Methods: Data sources for a description of the acronyms in the legend and the units.

is the catchability coefficient for the ith type of relative abundance index $I_{i, k}$ of species $k$. Because the units of $I_{i, k}$ are different (see Data Sources), the units of $q_{i, k}$ are different for different $i$. The unit of $I_{1, k}$ is per 10 million hook hour; the unit of $I_{2, k}$ is per thousand net per hour; the unit of $I_{3, k}$ is per thousand hooks; the unit of $I_{4, k}$ is per 100 thousand hooks per hour; the unit of $I_{5, k}$ is per 10 billion $\mathrm{m}^{2}$ per hour; and the unit of $I_{6, k}$ is per million hooks. Here we used the Schaefer model, $G_{k, t}=r_{k} N_{k, t}(1$ $-N_{k, t} / K_{k}$ ), as the production function, which is widely used in fisheries and ecology (May et al. 1979, Hilborn and Walters 1992), where $r_{k}$ is the population growth rate of species $k$ and $K_{k}$ is the carrying capacity of species $k$. Parameter $r_{k}$ was assumed to follow a normal distribution with mean $a_{1}=0.0661$ and variance $a_{2}=$ 0.0996. The values 0.0661 and 0.0996 are based on summarized information on the population growth rate of 80 shark species and stocks (Hoenig and Gruber 1990, Cailliet et al. 1992, Sminkey and Musick 1995, Smith et al. 1998, Cortés 2002a, Mollet and Cailliet
2002). The carrying capacity of each species, $K_{k}$, was assumed to follow a uniform distribution $(U)$ with lower bound $K_{\min , k}$ and upper bound $K_{\max , k}$. Maximum observed catch of species $k$ was used as the value of $K_{\min , k}$. The upper bound $K_{\max }$ was assumed to be the estimated carrying capacity of large coastal sharks (35 677000 sharks; NMFS 2006). The prior for $N_{1981}$, the abundance in the first year of our model, followed a lognormal distribution. We further assigned a uniform distribution between $1 \%$ and $100 \%$ of $K$ for the median of the prior for $N_{1981}$. The sensitivities of model results to these priors were explored (see Sensitivity analysis and Table 1).

\section{The hierarchical Bayesian surplus production model with species-specific data}

When the species-specific data were treated as hierarchically structured, instead of assessing each species separately, the three species were assessed together through a Bayesian hierarchical model. In the 
TABLE 1. Prior specification in seven scenarios used in sensitivity analyses.

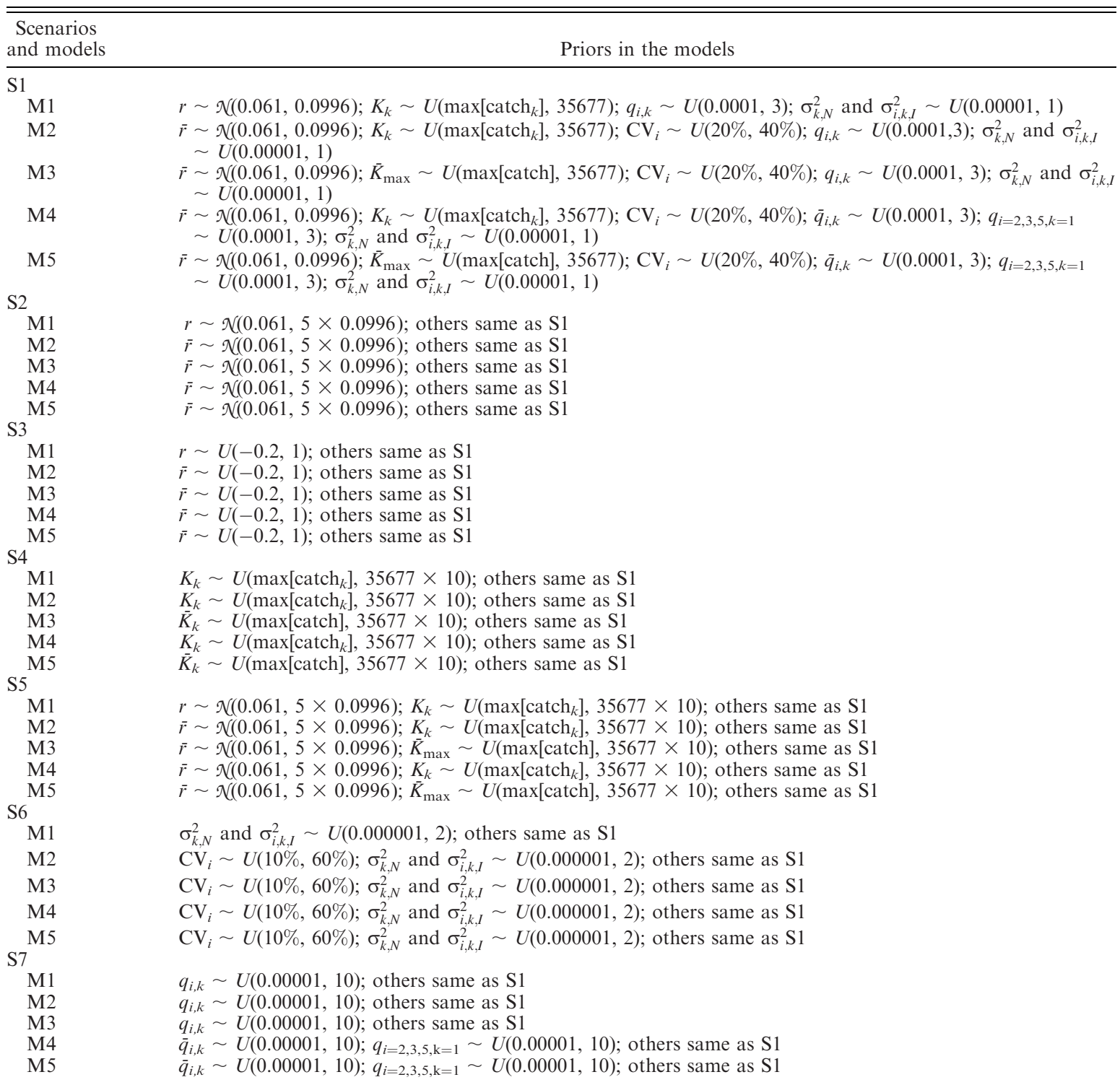

Notes: Each scenario included five models: M1, nonhierarchical priors; M2, hierarchical prior of growth rate, $r$; M3, hierarchical priors of $r$ and carrying capacity, $K$; M4, hierarchical priors of $r$ and catchability coefficient, $q$; M5, hierarchical priors of $r, K$, and $q$. $K$ values are in thousands. The subscript $k$ indicates the species; $i$ is the $i$ th type of relative abundance index, $I ; N$ is the abundance. $U(\max [\mathrm{catch}])$ is the uniform distribution of the maximum observed catch.

first hierarchical model, only $r$ was modeled to be hierarchically structured (M2)

$$
\begin{aligned}
G_{k, t} & =r_{k} N_{k, t}\left(1-N_{k, t} / K_{k}\right) \\
r_{k} & \sim \mathcal{N}\left(\bar{r},\left[\mathrm{CV}_{1} \times \bar{r}\right]^{2}\right) \\
\bar{r} & \sim \mathcal{N}\left(a_{1}, a_{2}\right) \\
\mathrm{CV}_{1} & \sim U\left(b_{1}, b_{2}\right) \\
K_{k} & \sim U\left(K_{\min , k}, K_{\max }\right) .
\end{aligned}
$$

Model M2 was used to simulate possible differences of the population growth rates of the three species in the complex via a hierarchical specification. The intrinsic rate of population growth, $r_{k}$, was assumed to follow a normal distribution with mean $\bar{r}$ and variance $\left(\mathrm{CV}_{1} \times\right.$ $\bar{r})^{2}$. However, mean $\bar{r}$ and coefficient of variation $\mathrm{CV}_{1}$ are hyperparameters. The parameter $\bar{r}$ itself was assumed to follow a normal distribution with mean $a_{1}$ $=0.0661$ and variance $a_{2}=0.0996$. The carrying capacity of each species, $K_{k}$, was assumed to follow a uniform distribution with lower bound $K_{\min , k}$ and upper bound $K_{\text {max }}$. Maximum observed catch of species $k$ was used as the value of $K_{\min , k}$. However, the upper bound $K_{\max }$ is 
the estimated carrying capacity of large coastal sharks (35 677000 sharks; NMFS 2006). Sensitivity of model results to these values and the hierarchical structure of $r$ was explored (see Sensitivity analysis). Values of CV of $20-40 \%$ have been treated as a reasonable range for fisheries data (Walters 1998); thus in the base scenario (S1), $\mathrm{CV}_{1}$ and $\mathrm{CV}_{2}$ were assumed to range between $20 \%$ and $40 \%$. We nevertheless tested the sensitivity of this assumption by extending the range to $10-60 \%$ in the sensitivity analysis.

In the second hierarchical model, both $r$ and $K$ were assumed to be with hierarchically structured priors (M3)

$$
\begin{aligned}
G_{k, t} & =r_{k} N_{k, t}\left(1-N_{k, t} / K_{k}\right) \\
r_{k} & \sim \mathcal{N}\left(\bar{r},\left[\mathrm{CV}_{1} \times \bar{r}\right]^{2}\right) \\
\bar{r} & \sim \mathcal{N}\left(a_{1}, a_{2}\right) \\
\mathrm{CV}_{1} & \sim U\left(b_{1}, b_{2}\right) \\
K_{k} & \sim U\left(K_{\min , k}, K_{\max , k}\right) \\
K_{\max , k} & \sim \mathcal{N}\left(\bar{K}_{\max },\left[\mathrm{C} V_{2} \times \bar{K}_{\max }\right]^{2}\right) \\
\bar{K}_{\max } & \sim U\left(c_{1}, c_{2}\right) \\
\mathrm{CV}_{2} & \sim U\left(d_{1}, d_{2}\right) .
\end{aligned}
$$

In this model, the carrying capacity of each species, $K_{k}$, was assumed to follow a uniform distribution with lower bound $K_{\min , k}$ and upper bound $K_{\max , k}$. Maximum observed catch of species $k$ was used as the value of $K_{\min , k}$. However, the upper bound $K_{\max , k}$ itself followed a two-level distribution with mean $\bar{K}_{\max }$, and variance, $\left(\mathrm{CV}_{2} \times \bar{K}_{\max }\right)^{2}$. The parameter $\bar{K}_{\max }$ was assumed to follow a uniform distribution with lower bound $c_{1}$, the maximum observed catch of large coastal sharks (1 200000 sharks) and upper bound, $c_{2}$, the estimated carrying capacity of large coastal sharks (35 677000 sharks; NMFS 2006). The use of $K_{\max , k} \sim \mathcal{N}\left(\bar{K}_{\max },\left[\mathrm{CV}_{2}\right.\right.$ $\left.\times \bar{K}_{\max }\right]^{2}$ ) incorporated uncertainty in the estimated carrying capacity of large coastal sharks into the prior information, compared with using an arbitrarily inflated prior for $K_{\max }$. Sensitivity of model results to these values and the hierarchical structure of $r, K, \mathrm{CV}_{1}$, and $\mathrm{CV}_{2}$ were explored (see Sensitivity analysis).

The surveys for relative abundance indices for great and smooth hammerheads were also available for scalloped hammerheads. In the fourth model (M4), both $r$ and $q_{i, k}$ was hierarchically structured to incorporate the potential similarity and relationship among catchability of the species $\left(q_{i, k}\right)$ if they appear in the same survey. According to the surveys and the species available in each survey, we can write the distributions of $q_{i, k}$ as follows:

$$
\begin{aligned}
q_{i=1,4, k=1,2} & \sim \mathcal{N}\left(\bar{q}_{i},\left[\bar{q}_{i} \times \mathrm{CV}_{3}\right]^{2}\right) \\
q_{i=6, k=1,3} & \sim \mathcal{N}\left(\bar{q}_{i},\left[\bar{q}_{i} \times \mathrm{CV}_{3}\right]^{2}\right) \\
q_{i=2,3,5, k=1} & \sim U\left(c_{i, 1}, c_{i, 2}\right) \\
\bar{q}_{i} & \sim U\left(c_{i, 1}, c_{i, 2}\right) \\
\mathrm{CV}_{3} & \sim U\left(e_{1}, e_{2}\right),
\end{aligned}
$$

where $c_{i, 1}$ and $c_{i, 2}$, the lower and upper bounds of $\bar{q}_{i}$, are assumed to be 0.0001 and $3 ; \mathrm{CV}_{3}$ is the $\mathrm{CV}$ of $q_{i, k}$ and is assumed to vary between $20-40 \%$ (i.e., limit $e_{1}=20 \%, e_{2}$ $=40 \%$ ). It also implied that for surveys in which only one species was observed (i.e., $i=2,3,5$ ), a nonhierarchical model was used, i.e., $q_{i=2,3,5, k=1}$ follows a uniform distribution with lower and upper bounds $c_{i, 1}$ and $c_{i, 2}$. Sensitivities to these prior values were tested (see Sensitivity analysis).

We also developed a model to allow $r, K$, and $q$ to be all hierarchically structured (M5), i.e., a hybrid of M3 and M4. Priors used in M3 and M4 were used in this model also. Sensitivities to these prior values were tested.

The observation-process-error estimator, which considers both the process error in the function of population size and the observation error in the function of abundance indices, was used in this study (Millar and Meyer 2000, de Valpine and Hastings 2002). Process error associated with the production and population dynamics equations is critically important given the simplicity of the production equation and potentially chaotic environmental changes. Recent research on comparison of the observation-error estimator and the observation-process-error estimator also suggests the importance of using the observation-process-error estimator (de Valpine and Hastings 2002, Calder et al. 2003).

A lognormal error structure was used for both the process and observation errors, i.e., the log-transformed population abundance $\ln \left(N_{k, t}\right)$ followed a normal distribution with mean $\ln \left(N_{k, t-1}+G_{k, t-1}-C_{k, t-1}\right)$ and variance $\sigma_{k, N}^{2}$; the log-transformed relative population abundance $\ln \left(I_{i, k, t}\right)$ followed a normal distribution with mean $\ln \left(q_{i, k} N_{k, t}\right)$ and variance $\sigma_{i, k, I}^{2}$. Priors for $\sigma_{k, N}^{2}$ and $\sigma_{i, k, I}^{2}$ were assumed to be uniform distributions between 0.00001 and 1 . To test the influence of these priors, $U(0.000001,2)$ was used in a sensitivity analysis.

\section{Bayesian approach and priors}

Presently only Bayesian methods are computationally possible for a hierarchically structured process-observation error model, and the traditional maximum likelihood methods cannot solve it unless some assumptions are made for the process error (de Valpine and Hastings 2002). The Bayesian approach uses a probability rule (Bayes' theorem) to calculate a "posterior distribution" 
from the observed data and a "prior distribution," which summarizes the prior knowledge of the parameters (Berger 1985, Gelman et al. 2004). The Bayesian approach was used to estimate both the uncertainty in parameter estimates and population abundance. A nonhierarchical Bayesian model for each species specifies a posterior density for parameters $(p(\theta \mid I))$ using Bayes' theorem. When species $k$ is considered, the posterior probability of all the parameters, represented by $\theta_{k}$, given observation of $I_{k}$ can be written as

$$
\begin{aligned}
p_{k}\left(\theta_{k} \mid I_{k}\right) \propto & L\left(I_{k} \mid N_{k}, q_{k}, \sigma_{k, I}\right) \pi\left(N_{k} \mid r_{k}, K_{k}, \sigma_{k, N}\right) \\
& \times \pi\left(q_{k}, \sigma_{k, I}\right) \pi\left(r_{k}, K_{k}, \sigma_{k, N}\right)
\end{aligned}
$$

where $L\left(I_{k} \mid N_{k}, q_{k}, \sigma_{k, I}\right)$ is the likelihood function of $I_{k}$ (all the available relative abundance indices for species $k$ ) and it is calculated as $L\left(I_{k} \mid N_{k}, q_{k}, \sigma_{k, I}\right)=\prod_{i} \prod_{t} g\left(I_{i, k, t} \mid N_{k, t}\right.$, $\left.q_{i, k}, \sigma_{i, k, I}\right)$. The expression $\pi\left(N_{k} \mid r_{k}, K_{k}, \sigma_{k, N}\right)$ is the joint distribution for $\left(N_{k, t}, t=1981, \ldots, 2005\right)$ and $\pi\left(N_{k} \mid r_{k}, K_{k}\right.$, $\left.\sigma_{k, N}\right)=\prod_{t} f\left(N_{k, t \geq 2} \mid N_{k, t-1}, r_{k}, K_{k}, \sigma_{k, N}\right) \pi\left(N_{k, 1}\right)$. In Eq. 5, $\pi\left(q_{k}, \sigma_{k, I}\right)=\prod_{i} \pi\left(q_{i, k}, \sigma_{i, k, I}\right)$.

$N_{k, 1}$ is assumed to follow a uniform distribution between $1 \%$ to $100 \%$ of $K$; The priors for $r_{k}, K_{k}, q_{i, k}$, $\sigma_{k, N}, \sigma_{i, k, I}, N_{k, 1}$ are assumed to be independent. Here, scalloped hammerhead shark $(k=1)$ has six indices $(i=$ $1, \ldots, 6)$; great hammerhead shark $(k=2)$ has two indices $(i=1,4)$; and smooth hammerhead shark has only one index $(k=3$ and $i=6)$.

A hierarchical Bayesian method assigns priors of hyperparameters to yield the joint posterior:

$$
\begin{aligned}
p\left(\theta^{\prime} \mid I_{i=1 \ldots 6, k=1 \ldots 3}\right) & \propto \prod_{k}\{ \\
\propto & L\left(I_{k} \mid N_{k}, q_{k}, \sigma_{k, I}\right) \pi\left(N_{k} \mid r_{k}, K_{k}, \sigma_{k, N}\right) \\
& \times h\left(N_{k, 1} \mid \bar{N}_{k, 1}, \sigma_{k, N}\right) \pi\left(r_{k} \mid \bar{r}, \mathrm{CV}_{1}\right) \pi\left(K_{k} \mid K_{\max , k}\right) \\
& \times \pi\left(K_{\max , k} \mid \bar{K}_{\max }, \mathrm{CV}_{2}\right) \pi\left(q_{i, k} \mid \bar{q}_{i}, \mathrm{CV}_{3}\right) \\
& \times \pi(\bar{r}) \pi\left(\bar{K}_{\max }\right) \pi\left(\mathrm{CV}_{1}\right) \pi\left(\mathrm{CV}_{2}\right) \pi\left(\mathrm{CV}_{3}\right) \pi\left(\bar{q}_{i}\right) \\
& \left.\times \pi\left(\sigma_{k, N}\right) \pi\left(\sigma_{i, k, I}\right) \pi\left(\bar{N}_{k, 1}\right)\right\} .
\end{aligned}
$$

In the above equations, $\theta^{\prime}=r_{k}, K_{k}, \sigma_{k, N}, \sigma_{i, k, I}, \bar{N}_{k, 1}, \bar{r}$, $\bar{K}_{\text {max }, k}, K_{\max }, q_{i, k}, \bar{q}_{i}, \mathrm{CV}_{1}, \mathrm{CV}_{2}, \mathrm{CV}_{3}$, and $k$ ranges from one to three species, and $i$ ranges from one to six relative abundance indices. $\bar{N}_{k, 1}$ is the median expectation of $N_{k, 1}$ and is assumed to follow a uniform distribution between $1 \%$ to $100 \%$ of $K_{k}$. The term $f\left(N_{k, t} \mid N_{k, t-1}, r_{k}, K_{k}, \sigma_{k, N}\right)$ is the probability density function (pdf) of $N_{k, t}$ given $N_{k, t-1}$ and parameters $r_{k}, K_{k}, \sigma_{k, N} ; g\left(I_{i, k, t} \mid N_{k, t}, q_{i, k}, \sigma_{i, k, I}\right)$ is the pdf of $I_{i, k, t}$ given variable $N_{k, t}$ and parameters $q_{i, k}, \sigma_{i, k, I} ; \pi\left(r_{k} \mid \bar{r}, \mathrm{CV}_{1}\right)$ is the pdf of $r_{k}$ given $\bar{r}$ and $\mathrm{CV}_{1}$; $\pi\left(K_{k} \mid K_{\max , k}\right)$ is the pdf of $K_{k}$ given $K_{\max , k}$; $\pi\left(K_{\max , k} \mid \bar{K}_{\max }, \mathrm{CV}_{2}\right)$ is the pdf of $K_{\max , k}$ given $\bar{K}_{\max }$,

\begin{tabular}{|c|c|c|c|c|c|c|}
\hline Scenario & Species & M1 & M2 & M3 & M4 & M5 \\
\hline S1 & $\begin{array}{l}\text { all } \\
\text { scalloped } \\
\text { great } \\
\text { smooth }\end{array}$ & $\begin{array}{r}117.47 \\
76.80 \\
35.86 \\
4.81\end{array}$ & 113.99 & 111.15 & 122.33 & 120.69 \\
\hline $\mathrm{S} 2$ & $\begin{array}{l}\text { all } \\
\text { scalloped } \\
\text { great } \\
\text { smooth }\end{array}$ & $\begin{array}{r}117.74 \\
76.89 \\
37.02 \\
3.83\end{array}$ & 112.09 & 113.40 & 121.00 & 121.09 \\
\hline S3 & $\begin{array}{l}\text { all } \\
\text { scalloped } \\
\text { great } \\
\text { smooth }\end{array}$ & $\begin{array}{r}117.21 \\
75.96 \\
36.92 \\
4.33\end{array}$ & 112.26 & 112.27 & 121.81 & 121.61 \\
\hline S4 & $\begin{array}{l}\text { all } \\
\text { scalloped } \\
\text { great } \\
\text { smooth }\end{array}$ & $\begin{array}{r}115.94 \\
76.38 \\
35.30 \\
4.26\end{array}$ & 108.73 & 109.56 & 120.95 & 120.80 \\
\hline S5 & $\begin{array}{l}\text { all } \\
\text { scalloped } \\
\text { great } \\
\text { smooth }\end{array}$ & $\begin{array}{r}121.67 \\
77.23 \\
38.81 \\
5.63\end{array}$ & 115.07 & 110.41 & 120.52 & 122.65 \\
\hline S6 & $\begin{array}{l}\text { all } \\
\text { scalloped } \\
\text { great } \\
\text { smooth }\end{array}$ & $\begin{array}{r}118.64 \\
76.31 \\
37.81 \\
4.52\end{array}$ & 116.74 & 116.05 & 122.81 & 122.16 \\
\hline S7 & $\begin{array}{l}\text { all } \\
\text { scalloped } \\
\text { great } \\
\text { smooth }\end{array}$ & $\begin{array}{r}117.47 \\
76.80 \\
35.86 \\
4.81\end{array}$ & 114.87 & 109.53 & 120.96 & 120.94 \\
\hline
\end{tabular}
$\mathrm{CV}_{2} ; \pi(\bar{r})$ and $\pi\left(\bar{K}_{\max }\right)$ are the pdf's of $\bar{r}$ and $\bar{K}_{\max } ; \pi\left(\bar{q}_{i}\right)$, $\pi\left(\mathrm{CV}_{1}\right), \pi\left(\mathrm{CV}_{2}\right), \pi\left(\mathrm{CV}_{3}\right), \pi\left(\sigma_{k, N}\right), \pi\left(\sigma_{k, I}\right)$, and $\pi\left(\bar{N}_{k, 1}\right)$ are the pdf's of $\bar{q}_{i}$ and $\mathrm{CV}_{s}(s=1,2,3), \sigma_{k, N}, \sigma_{i, k, I}$, and $\bar{N}_{k, 1}$.

We used WinBUGS, a numerically intensive software package that implements general Bayesian models using
TABLE 2. Goodness-of-fit for the five models expressed as deviance information criterion (DIC).

Note: Both total and species-specific DIC values are given for M1.

Markov chain Monte Carlo (MCMC) for model fitting (Gilks 1996, Millar and Meyer 2000, Spiegelhalter et al. 2004). The computing code is available in the Supplement.

A critical issue in using MCMC methods is how to determine when random draws have converged to the posterior distribution. Here, three methods were used: monitoring the trace for key parameters, diagnosing the autocorrelation plot for key parameters, and using the Gelman and Rubin statistic (Gelman and Rubin 1992, Spiegelhalter et al. 2004). A detailed description of the use of these methods in fisheries can be found in $\mathrm{Su}$ et al. (2001). In this study, three chains were used. The three chains converged after 50000 iterations with a thinning interval of 5 based on the convergence criteria and were discarded. A thinning interval of 5 was then used to avoid parameter autocorrelation and another 20000 iterations were run to generate the posterior distributions. The posterior distributions of the key parameters were obtained through a kernel smoothing approach available in MATLAB (Bowman and Azzalini 1997, MathWorks 2010).

\section{Sensitivity analysis}

The sensitivity of the model outcomes to the specified priors was tested through a comparison of the informative prior of $\bar{r}$ in the hierarchical models (M2 and M3) and the $r$ in the nonhierarchical models (M1; Tables 1 and 2). In scenario 1 of the hierarchical models, the 
TABle 3. Posterior estimates of $r$ and $K$ under seven scenarios for each of five Bayesian state-space surplus production models and hammerhead shark species.

\begin{tabular}{|c|c|c|c|c|}
\hline Model and species & $\mathrm{S} 1$ & S2 & S3 & S4 \\
\hline \multicolumn{5}{|l|}{ M1 } \\
\hline $\begin{array}{l}\text { Scalloped } \\
\text { Great } \\
\text { Smooth }\end{array}$ & $\begin{array}{l}r(0.10,0.34,0.68) \\
K(99,215,1136) \\
r(-0.06,0.17,0.56) \\
K(187,487,3155) \\
r(-0.11,0.15,0.56) \\
K(28,89,677)\end{array}$ & $\begin{array}{l}r(0.15,0.43,0.88) \\
K(83,179,597) \\
r(-0.05,0.22,0.79) \\
K(171,435,2820) \\
r(-0.10,0.20,0.70) \\
K(26,82,352)\end{array}$ & $\begin{array}{c}r(0.14,0.46,0.93) \\
\mathrm{K}(81,159,578) \\
r(-0.04,0.24,0.84) \\
K(161,416,3137) \\
r(-0.11,0.18,0.83) \\
K(23,90,663)\end{array}$ & $\begin{array}{l}r(0.10,0.33,0.66) \\
K(101,233,1177) \\
r(-0.05,0.18,0.57) \\
K(187,486,2821) \\
r(-0.11,0.15,0.56) \\
K(30,89,457)\end{array}$ \\
\hline \multicolumn{5}{|l|}{ M2 } \\
\hline $\begin{array}{l}\text { Scalloped } \\
\text { Great } \\
\text { Smooth }\end{array}$ & $\begin{array}{l}r(0.07,0.24,0.53) \\
K(111,246,1150) \\
r(0.05,0.19,0.47) \\
K(194,374,1533) \\
r(0.05,0.20,0.47) \\
K(28,57,224)\end{array}$ & $\begin{array}{l}r(0.01,0.24,0.58) \\
K(110,251,1145) \\
r(0.01,0.19,0.51) \\
K(187,364,2318) \\
r(0.01,0.20,0.51) \\
K(27,58,301)\end{array}$ & $\begin{array}{l}r(0.09,0.25,0.54) \\
K(110,238,1363) \\
r(0.05,0.20,0.51) \\
K(195,381,2113) \\
r(0.06,0.21,0.52) \\
K(28,61,254)\end{array}$ & $\begin{array}{l}r(0.08,0.24,0.49) \\
K(109,202,977) \\
r(0.06,0.19,0.45) \\
K(199,394,2341) \\
r(0.05,0.20,0.44) \\
K(29,60,274)\end{array}$ \\
\hline \multicolumn{5}{|l|}{ M3 } \\
\hline $\begin{array}{l}\text { Scalloped } \\
\text { Great } \\
\text { Smooth }\end{array}$ & $\begin{array}{c}r(0.08,0.24,0.51) \\
K(112,227,1010) \\
r(0.05,0.19,0.46) \\
K(199,409,1575) \\
r(0.06,0.19,0.49) \\
K(28,62,231)\end{array}$ & $\begin{array}{c}r(0.08,0.26,0.53) \\
K(111,214,1036) \\
r(0.06,0.21,0.50) \\
K(195,356,1268) \\
r(0.06,0.20,0.48) \\
K(28,57,229)\end{array}$ & $\begin{array}{c}r(0.07,0.27,0.57) \\
K(108,203,962) \\
r(0.05,0.21,0.53) \\
K(183,382,1402) \\
r(0.05,0.21,0.50) \\
K(28,62,246)\end{array}$ & $\begin{array}{c}r(0.07,0.24,0.50) \\
K(119,245,1204) \\
r(0.05,0.18,0.46) \\
K(200,428,1855) \\
r(0.05,0.20,0.46) \\
K(29,62,251)\end{array}$ \\
\hline \multicolumn{5}{|l|}{ M4 } \\
\hline $\begin{array}{l}\text { Scalloped } \\
\text { Great } \\
\text { Smooth }\end{array}$ & $\begin{array}{c}r(0.05,0.20,0.43) \\
K(123,247,1418) \\
r(0.04,0.17,0.41) \\
K(112,236,1299) \\
r(0.03,0.15,0.39) \\
K(34,74,339)\end{array}$ & $\begin{array}{c}r(0.05,0.20,0.42) \\
K(130,242,769) \\
r(0.04,0.16,0.44) \\
K(112,253,1786) \\
r(0.03,0.14,0.40) \\
K(33,80,534)\end{array}$ & $\begin{array}{c}r(0.03,0.19,0.44) \\
\mathrm{K}(118,252,1141) \\
r(0.03,0.16,0.42) \\
K(116,250,1194) \\
r(0.02,0.14,0.37) \\
K(35,86,323)\end{array}$ & $\begin{array}{c}r(0.05,0.18,0.39) \\
K(130,285,1303) \\
r(0.04,0.16,0.38) \\
K(120,265,1135) \\
r(0.02,0.14,0.35) \\
K(35,90,423)\end{array}$ \\
\hline \multicolumn{5}{|l|}{ M5 } \\
\hline $\begin{array}{l}\text { Scalloped } \\
\text { Great } \\
\text { Smooth }\end{array}$ & $\begin{array}{c}r(0.06,0.20,0.41) \\
K(123,250,1062) \\
r(0.04,0.16,0.40) \\
K(116,256,1472) \\
r(0.04,0.14,0.33) \\
K(37,86,378)\end{array}$ & $\begin{array}{c}r(0.06,0.20,0.44) \\
K(128,263,886) \\
r(0.05,0.16,0.40) \\
K(117,250,1174) \\
r(0.03,0.15,0.38) \\
K(37,88,507)\end{array}$ & $\begin{array}{c}r(0.06,0.20,0.44) \\
\mathrm{K}(124,303,1400) \\
r(0.05,0.16,0.40) \\
K(118,267,1470) \\
r(0.03,0.15,0.38) \\
K(35,75,276)\end{array}$ & $\begin{array}{c}r(0.05,0.18,0.42) \\
K(125,265,1320) \\
r(0.04,0.16,0.40) \\
K(110,243,1379) \\
r(0.03,0.14,0.36) \\
K(36,89,421)\end{array}$ \\
\hline
\end{tabular}

Notes: Values in parentheses are the $2.5 \%$ percentile, median, and $97.5 \%$ percentile. $K$ values are in thousands.

hyperprior distribution of $\bar{r}$ was from the meta-analysis of 80 shark species and stocks; in scenario 2, the hyperparameter of variance in the hyperprior distribution was five times that from the meta-analysis $(\bar{r} \sim$ $\left.N\left(a_{1}, 5 \times a_{2}\right)\right)$; in scenario $3, \bar{r}$ was assumed to be between -0.2 and 1 . The fourth scenario consisted of increasing the upper bound of $\bar{K}_{\max }$ to 356770000 , i.e., 10 times the estimated carrying capacity of large coastal sharks. Our fifth scenario was to increase the variance of $\bar{r}$ five times and the upper bound of $\bar{K}_{\max }$ to 356770000 . Our sixth scenario involved widening $\mathrm{CV}_{s}, \sigma_{k, N}$, and $\sigma_{i, k, I}$, and the seventh scenario was to widen $\bar{q}_{i}$ in the hierarchical Bayesian models to a uniform distribution $U(0.00001,10)$, or $q_{i}$ if $q_{i}$ is not hierarchically structured to $U(0.00001,10)$.

\section{Model goodness-of-fit and model selection}

The goodness-of-fit of each of the Bayesian hierarchical surplus production models was compared to that of the traditional nonhierarchical surplus production model based on the values of the DIC:

$$
\begin{aligned}
\mathrm{DIC} & =2 \bar{D}-\hat{D}=\bar{D}+p_{D} \\
D(y, \theta) & =-2 \log \operatorname{likelihood}(y \mid \theta) \\
p_{D} & =\bar{D}-\hat{D}
\end{aligned}
$$

where $D$ is deviance, a measurement of prediction goodness for our models, $p_{D}$ is the effective number of parameters in a Bayesian model, $\bar{D}$ is the posterior mean of the deviance, and $\hat{D}$ is the deviance of the posterior mean. Here, $y$ is $I_{i, k}$ and $\theta$ includes all the parameters in the corresponding models. The DIC is a hierarchical modeling generalization of the AIC (Akaike information criterion) and $\mathrm{BIC}$ (Bayesian information criterion, also known as the Schwarz criterion). It is particularly useful in Bayesian model selection problems, where the posterior distributions of the models have been obtained by MCMC simulation (Spiegelhalter et al. 2002, 2004). The DIC values for the nonhierarchical models (M1) were computed as the sum of the DIC values from each individual species-specific model. 
TABLE 3. Extended.

\begin{tabular}{lll}
\hline \hline S5 & S6 & S7 \\
\hline
\end{tabular}

$r(0.13,0.42,0.88) \quad r(0.11,0.37,0.71) \quad r(0.10,0.34,0.68)$

$K(86,165,580) \quad K(92,176,668) \quad K(99,215,1136)$

$r(-0.04,0.23,0.79) \quad r(-0.05,0.18,0.56) \quad r(-0.05,0.17,0.56)$

$K(167,396,2024) \quad K(190,480,2596) \quad K(187,487,3155)$

$r(-0.11,0.17,0.69) \quad r(-0.11,0.15,0.55) \quad r(-0.11,0.15,0.56)$

$K(26,96,555) \quad K(29,97,532) \quad K(28,89,677)$

$r(0.07,0.26,0.60) \quad r(0.08,0.25,0.51) \quad r(0.07,0.24,0.53)$

$K(109,212,772) \quad K(114,212,879) \quad K(111,246,1150)$

$r(0.05,0.20,0.50) \quad r(0.04,0.19,0.44) \quad r(0.05,0.19,0.47)$

$K(191,365,1541) \quad K(199,376,1754) \quad K(194,374,1533)$

$r(0.05,0.21,0.51) \quad r(0.03,0.18,0.44) \quad r(0.05,0.20,0.47)$

$K(28,58,276) \quad K(30,62,262) \quad K(28,57,224)$

$r(0.09,0.26,0.57)$

$K(109,224,894)$

$r(0.06,0.21,0.1)$

$K(192,375,1366)$

$r(0.06,0.21,0.51)$

$K(29,57,234)$

$r(0.04,0.22,0.52)$ $K(123,260,1409)$

$r(0.02,0.18,0.46)$

$K(195,372,1277)$

$r(0.01,0.19,0.46)$

$K(29,61,256)$

$r(0.05,0.21,0.47)$

$K(118,249,1066)$

$r(0.04,0.17,046)$

$K(107,237,1340)$

$r(0.03,0.16,0.39)$

$K(35,74,319)$

$r(0.05,0.19,0.40)$

$K(132,260,1340)$

$r(0.04,0.16,0.38)$

$K(115,256,1207)$

$r(0.03,0.14,0.36)$

$K(35,82,390)$

$r(0.04,0.20,0.46)$ $K(118,259,1032)$ $r(0.03,0.17,0.48)$ $K(105,237,2222)$

$r(0.00,0.13,0.37)$ $K(36,86,378)$

$r(0.05,0.18,0.39)$ $K(122,255,1189)$ $r(0.04,0.15,0.43)$ $K(114,245,1160)$ $r(0.03,0.14,0.36)$ $K(36,87,397)$

$r(0.08,0.24,0.51)$ $K(112,227,1010)$ $r(0.05,0.19,0.46)$ $K(199,409,1575)$ $r(0.06,0.19,0.49)$ $K(28,62,231)$

$r(0.05,0.18,0.40)$ $K(132,268,1117)$ $r(0.04,0.16,0.40)$ $K(116,250,16923)$ $r(0.03,0.14,0.36)$ $K(36,84,439)$

$r(0.05,0.18,0.40)$ $K(128,265,1213)$ $r(0.04,0.15,0.40)$ $K(115,254,1453)$ $r(0.03,0.14,0.35)$ $K(38,78,350)$

Model robustness is also often considered when comparing hierarchical and nonhierarchical models (Roberts and Rosenthal 2001) because model selection based on fit can only be inconclusive, especially when differences in DIC values are less than 5 (Spiegelhalter et al. 2002, Jiao et al. 2008, 2009b). We considered both model robustness and fit in our study because of the limited number of groups in the hierarchical model (3).

Under the assumption of a Schaefer population growth model, $N_{\mathrm{msy}}=r K / 4$ is the abundance at maximum sustainable yield (msy) and $F_{\mathrm{msy}}=r / 2$ is the rate of fishing mortality at msy. The estimated rate of fishing mortality $F$ relative to $F_{\mathrm{msy}}, F / F_{\mathrm{msy}}$, and population size relative to $N_{\text {msy }}, N / N_{\text {msy }}$, were also compared among different models. The probability of $F$ being larger than $F_{\mathrm{msy}}$, i.e., $P\left(F>F_{\mathrm{msy}}\right)$ was used to define the risk of overfishing. It indicates the exploitation status of the population. The probability of $N$ being smaller than $N_{\text {msy }}$, i.e., $P\left(N<N_{\text {msy }}\right)$ was used to define the risk of a population being overfished. It is a measure of the depletion level of the population and its present status in terms of abundance.

\section{RESULTS}

Hierarchical models with a two-level prior of $r$ (M2) and two-level priors of $r$ and $K$ (M3) yielded smaller DIC values (108-116) than nonhierarchical models (M1; 115-122; Table 2). However, hierarchical models with $r$ and $q$ (M4) or $r, K$, and $q$ hierarchically structured (M5) resulted in larger DIC values (120-123) than for M1, M2, and M3 (Table 2), thus consistently yielding the worst fit of the five models tested. The DIC values and parameter estimates can be influenced by the choice of priors as reflected by their variation among sensitivity scenarios (Tables 2 and 3). Although there were differences among DICs the differences were limited between M2 and M3.

The hierarchical models were very robust to the hyperparameters. Parameters $r$ and $K$ varied very little when the variance of the hyperparameter of $r$ was multiplied by factors of 5 (scenario 2) with respect to that used in scenario 1 . The nonhierarchical models were less robust than the hierarchical models (Fig. 3). Similar results were found when the distribution of $\bar{r}$ was less informative, with a wide uniform distribution (scenario 3) and when both the variance of the hyperparameter of $r$ and the upper bound of $\bar{K}_{\text {max }}$ were increased by a factor of 5 and 10 , respectively (scenario 5). Results seemed less sensitive to changes in the upper bound of $\bar{K}_{\text {max }}$ (scenarios 2, 4 and 5). Results from scenario 4 were close to those of scenario 1 even though the upper bound of the prior $\bar{K}_{\max }$ increased by a factor of 10 (Table 3 and Fig. 3).

When model M1 was used, a less informative distribution of $r$ resulted in higher posterior values of $r$ and lower posterior values of $K$ (scenarios 2, 3, and 5; Table 3; Fig. 3). Less informative prior distributions of $K$ (scenarios 4 and 5), or $q_{i}$ (scenario 7), did not substantially influence results. However, all of the hierarchical models were very robust to the less informative distributions of both $r$ and $K$ (Table 3; Fig. 3).

Models M2 and M3 resulted in similar results in both parameters and population size estimation; while models M4 and M5 resulted in similar results but the results were somewhat different compared to these from M2 and M3 (Figs. 3 and 4). Models M2 and M3 resulted in higher posterior $r$ values for these three species than when using M4 or M5 (Table 3; Fig. 3A). The posterior of $K$ for great hammerhead was noticeably lower for M4 and M5 than for M1 to M3 (Table 3; Fig. 3B). The posterior population size estimates for great hammerhead shark were lower when M4 and M5 were used than when M2 and M3 were used (Fig. 4).

When $K_{k}$ was not hierarchically structured (M2), the resulting DIC values were close to those for the hierarchical structured $K_{k}(\mathrm{M} 3)$, and model results were very similar. Increasing the upper bound of $K_{k}$, i.e., $K_{\max , k}$ from $c_{2}$ to 10 times the value of $c_{2}$ did not change appreciably the estimates of $r$ or $K$. This indicated that the estimated carrying capacity of large coastal sharks (NMFS 2006) is a large enough quantity to be the upper bound of $K_{k}$. 
A
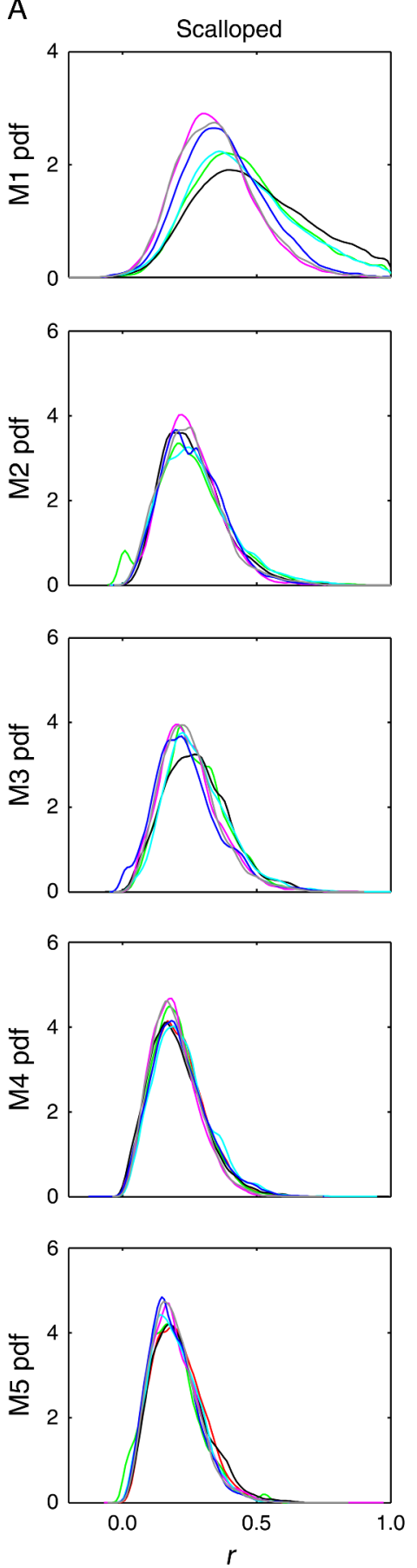

Great
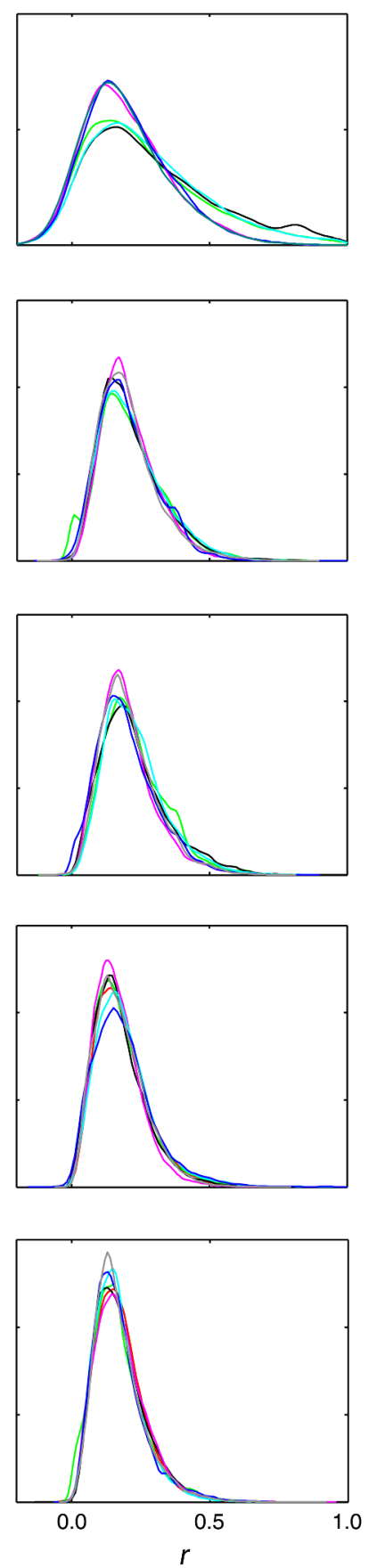

Smooth
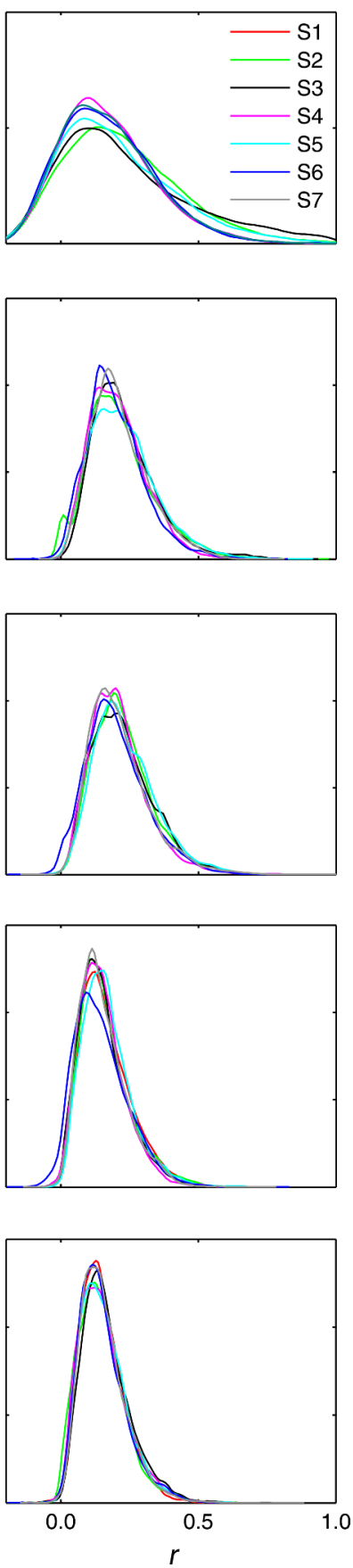

FIG. 3. Probability density functions (pdf's) of (A) growth rate $(r)$ and (B) carrying capacity $(K)$ from the five models under seven scenarios (S1-S7) for the three hammerhead shark species.

The three methods we used failed to show evidence against convergence of the MCMC algorithm. The Gelman and Rubin statistic for all parameters, including all variance terms, ranged from 0.95 to 1.01 , indicating convergence of the Markov chains. The autocorrelation function plot indicated a thinning interval of 5 , which was large enough to address potential autocorrelation in the MCMC runs (figures are not shown). We also visually observed the trace plots of the major parameters, which showed good mixing of the three chains, also indicative of convergence of the MCMC chains.

The Bayesian hierarchical state-space surplus production models, M2 to M4, fitted the data reasonably well (Fig. 5). The population of scalloped hammerhead likely 
B
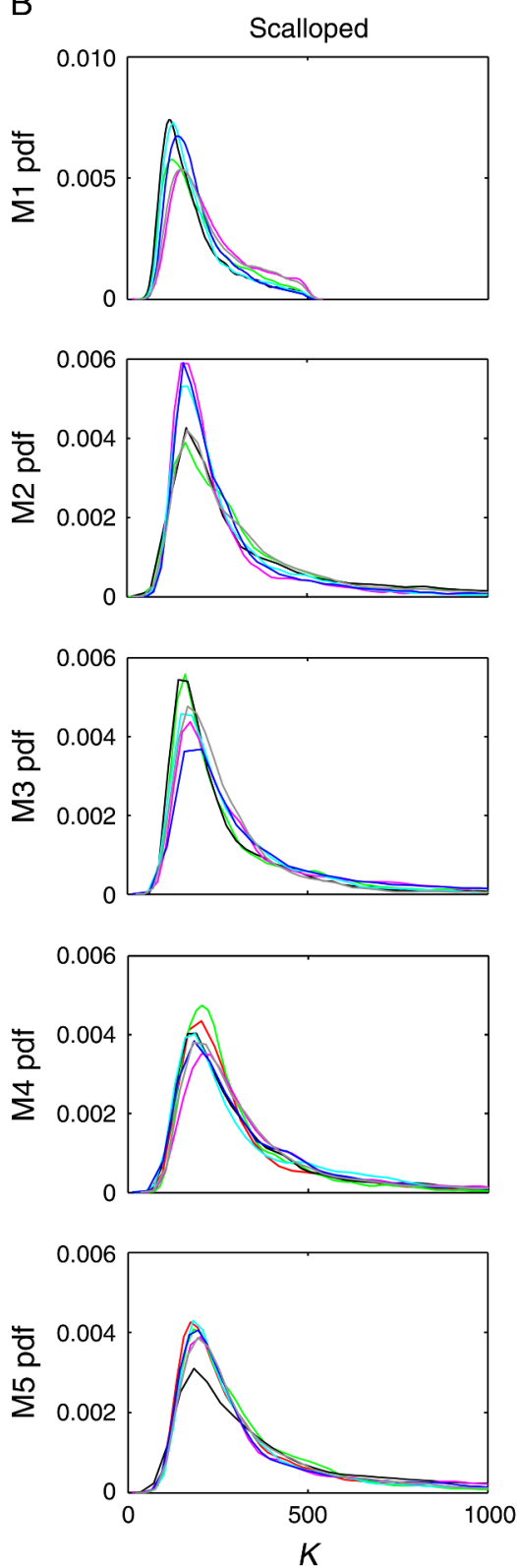
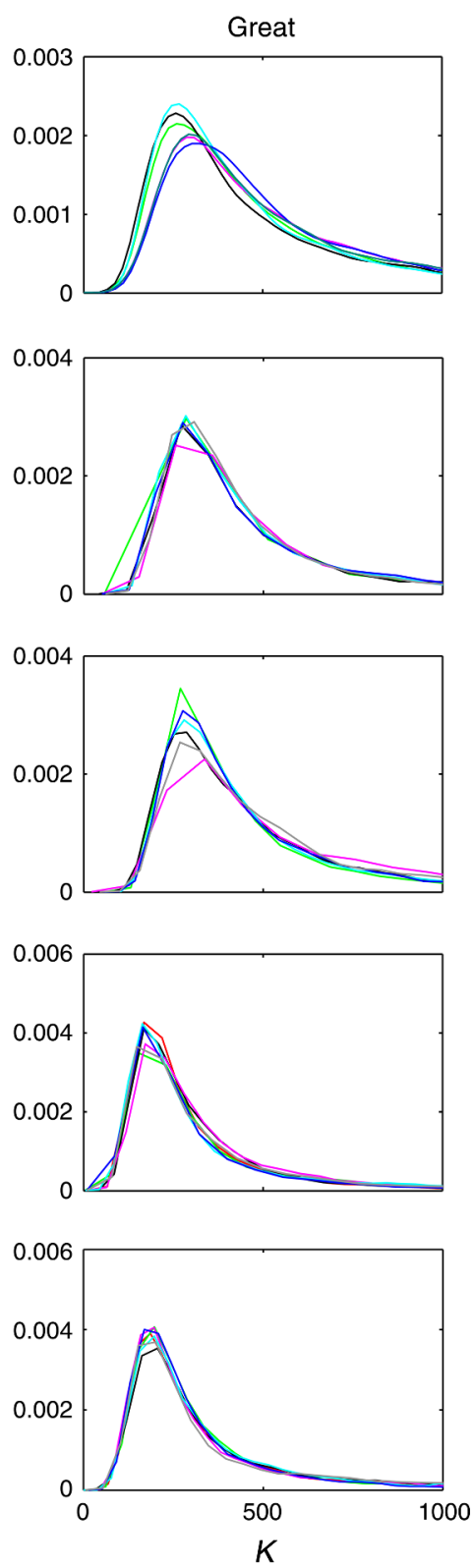

FIG. 3. Continued.
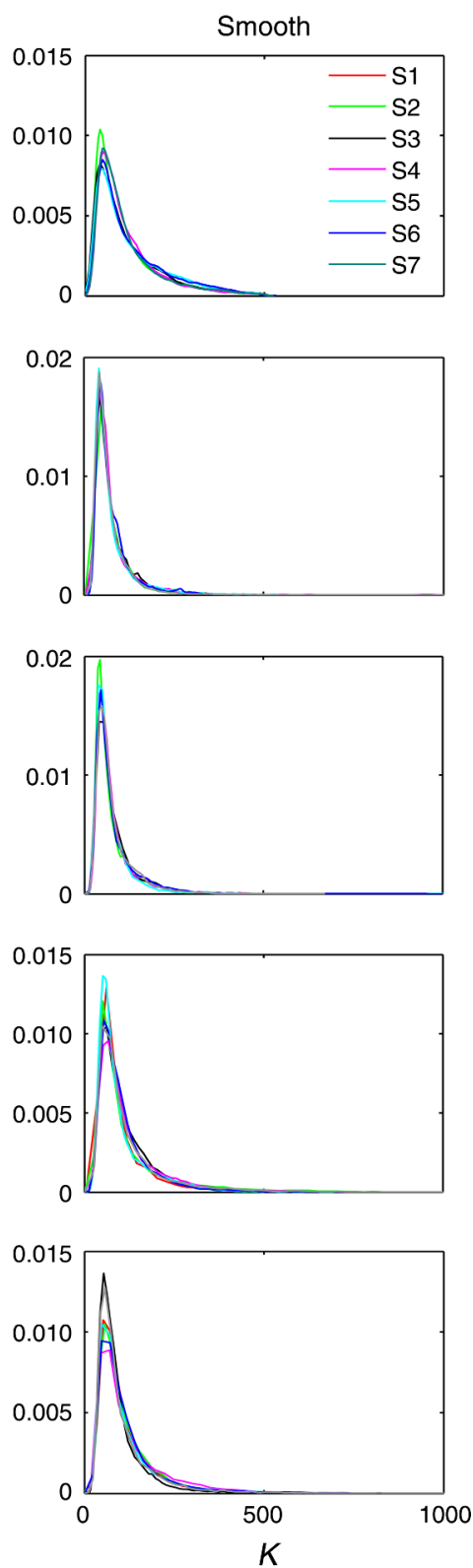

became overfished from the early 1980s and overfishing occurred periodically from 1983 to 2005; the risk of overfishing was still high in recent years (Fig. 6). The population of great hammerhead likely became overfished in the mid-1980s and overfishing occurred periodically from 1983 to 1997; the risk of overfishing was very low after 2001 (Fig. 6). The population of smooth hammerhead likely become overfished since mid-1980s but the degree of overfishing was not as high that for scalloped hammerhead sharks until the mid1990s and overfishing occurred continuously in 19941998 , but the risk of overfishing was generally very low after 2001 (Fig. 6).
Discussion

The goodness of fit (DIC values) of the hierarchical models M2 to M3 were the lowest and the differences between them were very limited. The DICs of M4 and M5 were higher than that of the nonhierarchical model (M1) for all three species considered, likely because of the limited number of species (three) hierarchically structured in our study. The limited differences in DIC values between M2 and M3, and between M4 and M5, prescribed the need for using multiple criteria to compare model performance instead of using information-based criteria only. Use of less informative priors 

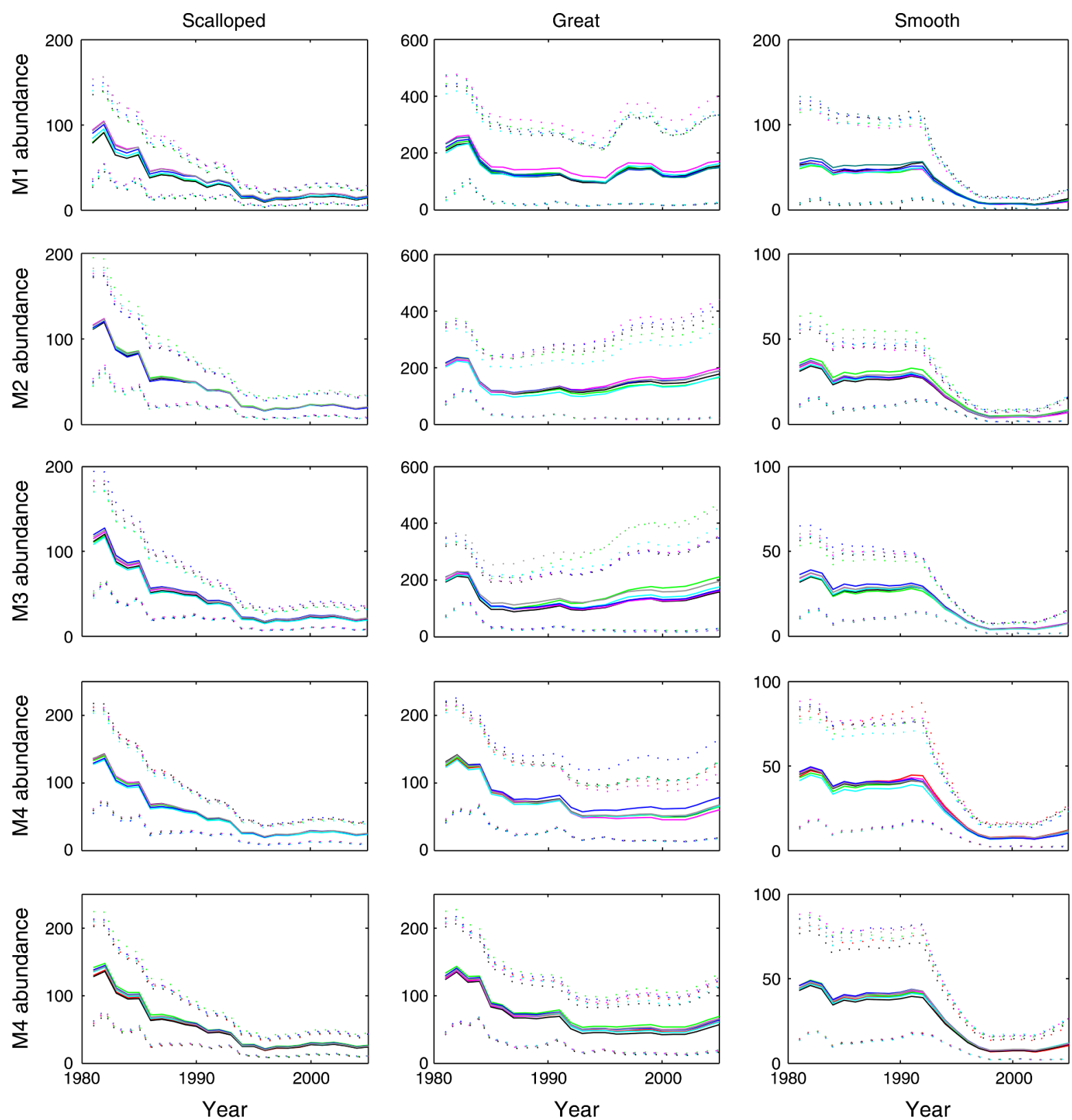

FIG. 4. Population abundance trajectories (in thousands of fish) for scalloped, great, and smooth hammerhead sharks. Solid lines denote the posterior mean of population abundance; dotted lines denote $95 \%$ credible intervals of population abundance. Different scenarios are denoted by different colors, which have the same meaning as in Fig. 3.

for the means of $r, K$, or $q$ and associated variances consistently yielded robust results with the hierarchical models. The robustness of the results indicated that even when using uninformative priors with a limited number of species, the hierarchical models were still useful and are a convenient approach for data-poor species to borrow strength from more data-rich species. A recent study on protein interactions based on a likelihood approach and three species also indicated the advantages of using multiple organisms and hierarchical models (Liu et al. 2005).
The sensitivity analyses conducted showed that hierarchical Bayesian models (with a multilevel prior of $r, r$ and $q, r$ and $K$, or $r, K$, and $q$ ) provided a more robust fit to the hammerhead data than nonhierarchical models. This suggests that a hierarchical Bayesian model or the use of multilevel priors is mathematically viable and valuable in modeling these three hammerhead shark populations and potentially other fish complexes for which species-specific data are available. However, this study also revealed that informative priors influence the results of both model parameters 

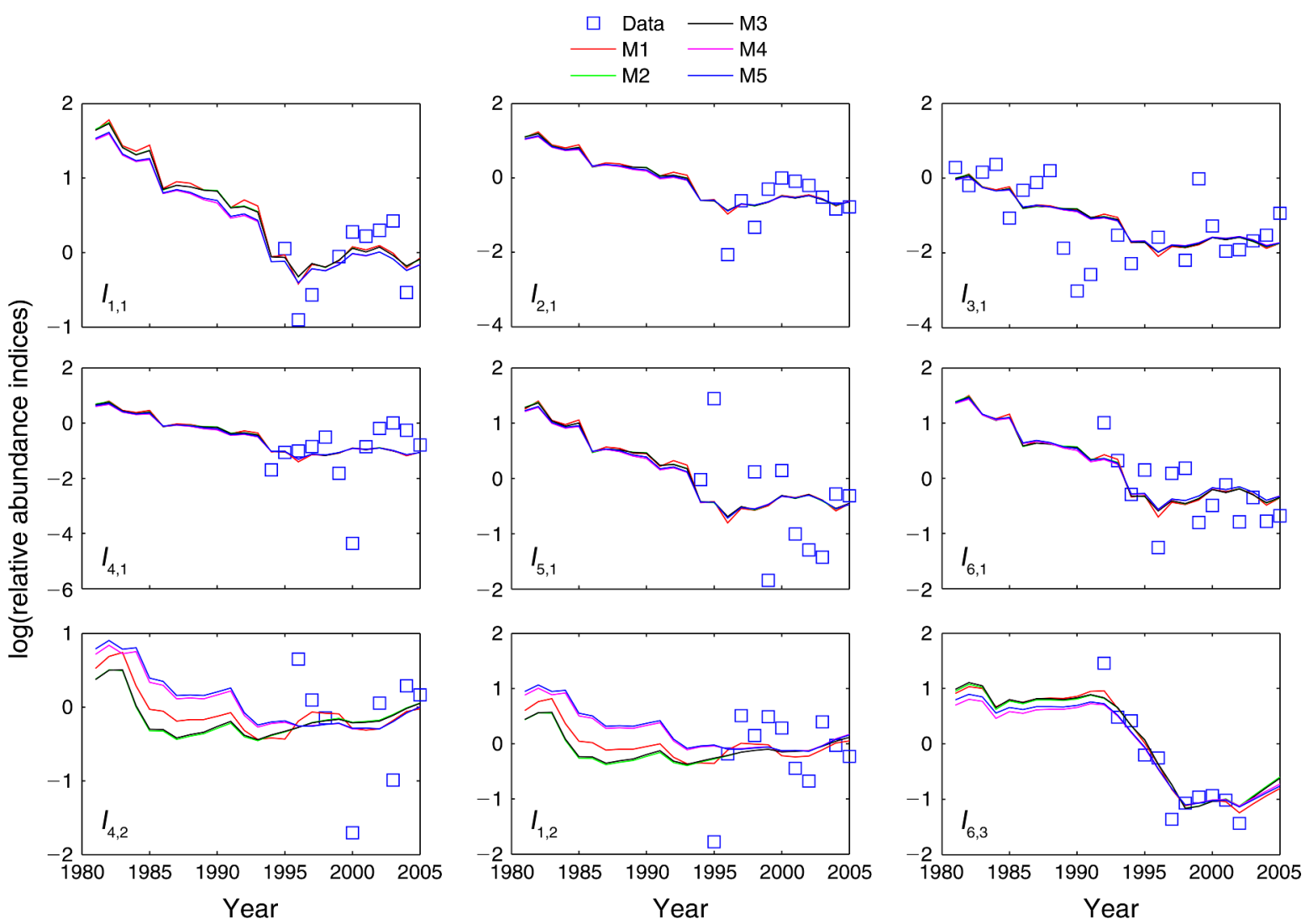

Fig. 5. Model fits to the logarithm of relative abundance indices from the five models under scenario 1. See Methods: Bayesian state-space surplus production model for each species for description of indices $I_{i, k}$.

and model fit (DIC values). The use of informative priors and model choice may also need to be justified based on an understanding of the biology of each species in the fish complex. In all, both the model goodness-of-fit and the fact that the Bayesian hierarchical model with a multilevel prior of $r$ (M2) resulted in robust results for all three species in the complex suggest that this model is a better choice.

Although the results from M4 and M5 with $r$ and $q$ or $r, K$, and $q$ hierarchically structured were also robust to prior specification, the DIC values were higher than those of the nonhierarchical models (M1). This suggests that M4 and M5 were not as good a choice as M2, possibly because of lack of informative priors on $q_{i}$. However, although DIC values of M4 an M5 were larger, the pdf profiles were narrower and modeling results for great and smooth hammerheads tended to be more biologically reasonable, with M4 and M5 estimating smaller population growth rates than M2 and M3. Although there have been few studies on the biology of great and smooth hammerheads, it is likely that the two species have population growth rates that differ substantially from those of scalloped hammerheads (E. Cortés, unpublished data). Future studies that allow specification of informative priors are thus recommended. In addition to gaining a better under- standing of the biology of these three species and specifying more informative priors on catchability $(q)$, Bayesian model averaging could be used as an alternative method that would allow model M4 and M5 to still be considered given that it was robust and the DIC values were not much larger than that of the nonhierarchical model (M1) (Spiegelhalter et al. 2002, Jiao et al. 2008, 2009b).

Multilevel priors are robust priors given the stability of the model results (Roberts and Rosenthal 2001). Our study showed consistent results across seven scenarios with multilevel priors. Compared with the commonly used priors, multilevel priors therefore may be better choices as robust priors. The number of levels of priors will depend on the parameters of interest and biological reality. For example, here we were interested in the population growth rate $r$ rather than hyperparameter $\bar{r}$; hence we had two levels of hyperparameters, which resulted in a stable distribution of $r$. We were also aware that the mean of $r_{k}$ can be different among species. Thus, a multilevel prior of $r$ reflects the biology of the fish species and allows the data-poor species to borrow strength from data-rich species. The resulting distributions of $r_{k}$ for both datarich and data-poor species were more stable and showed uncertainty reduction when hierarchical mod- 
A

Scalloped
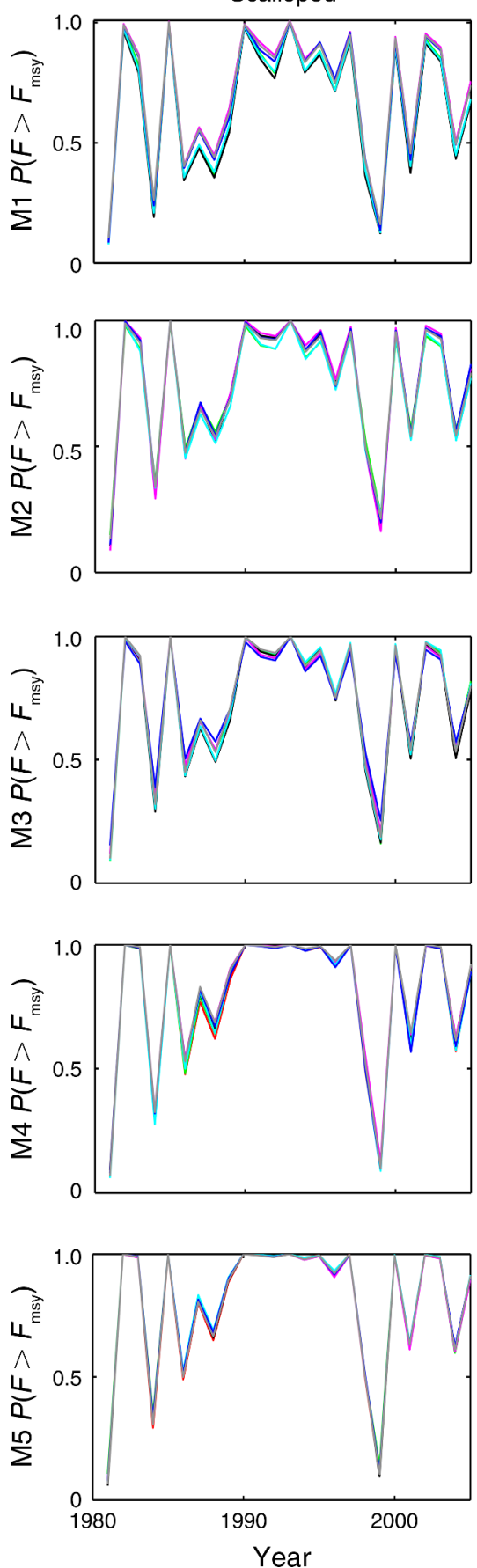

Great
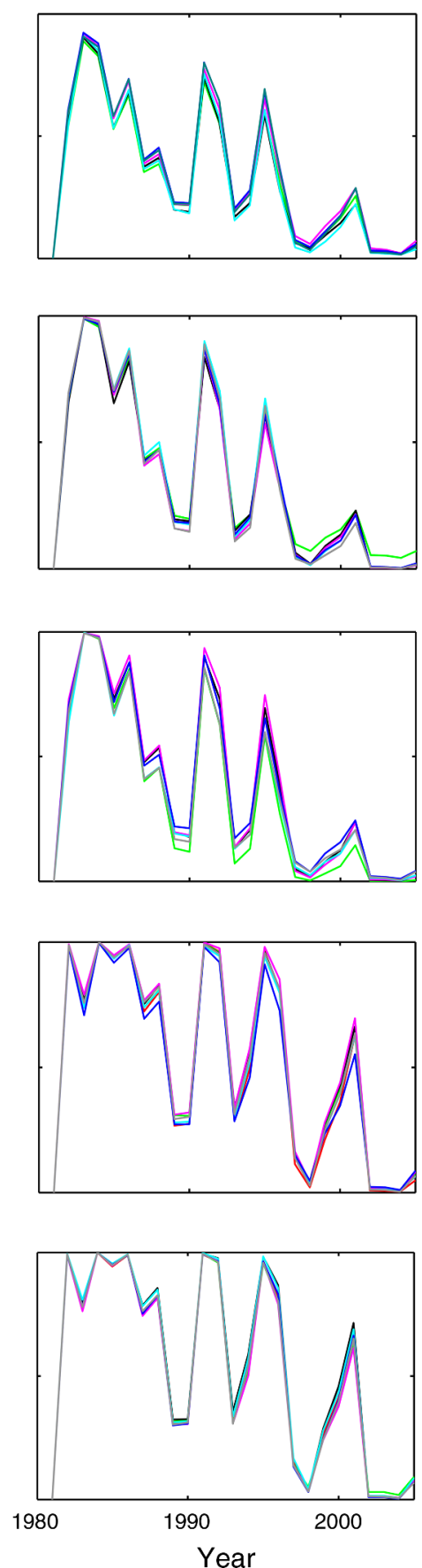

Smooth
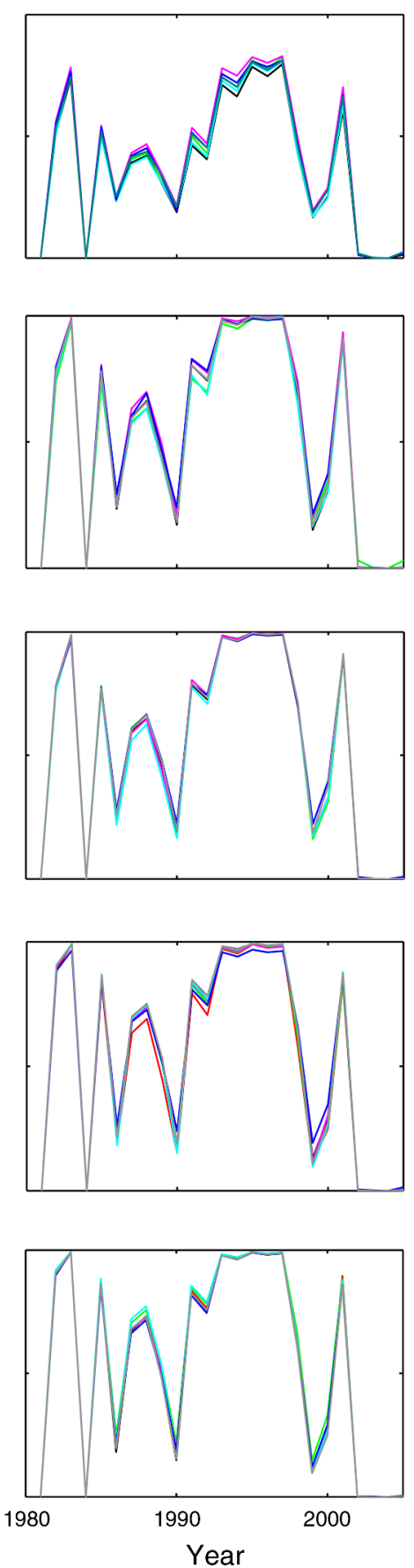

FIG. 6. (A) Probability of fishing mortality, $F$, being larger than $F_{\text {msy }}$ and (B) probability of population size, $N$, being smaller than $N_{\text {msy }}$ for the three hammerhead shark species under seven scenarios, where the subscript msy denotes the value at maximum sustainable yield. Different scenarios are denoted by different colors, which are the same as in Fig. 3.

els were used. This indicated that the hierarchical models benefit not just the data-poor species but also the data-rich species.

It is a common perception that MCMC output should be thinned to reduce autocorrelation (Gelman et al. 2004, Spiegelhalter et al. 2004). However, to estimate posterior quantities, it has been proven that thinning is suboptimal (MacEachern and Berliner 1994). We reran the models and scenarios with a thinning interval of 2 and 0 , respectively, but the results were the same as with a thinning interval of 5. Although most Bayesian analyses still use thinning in MCMC to eliminate 
B
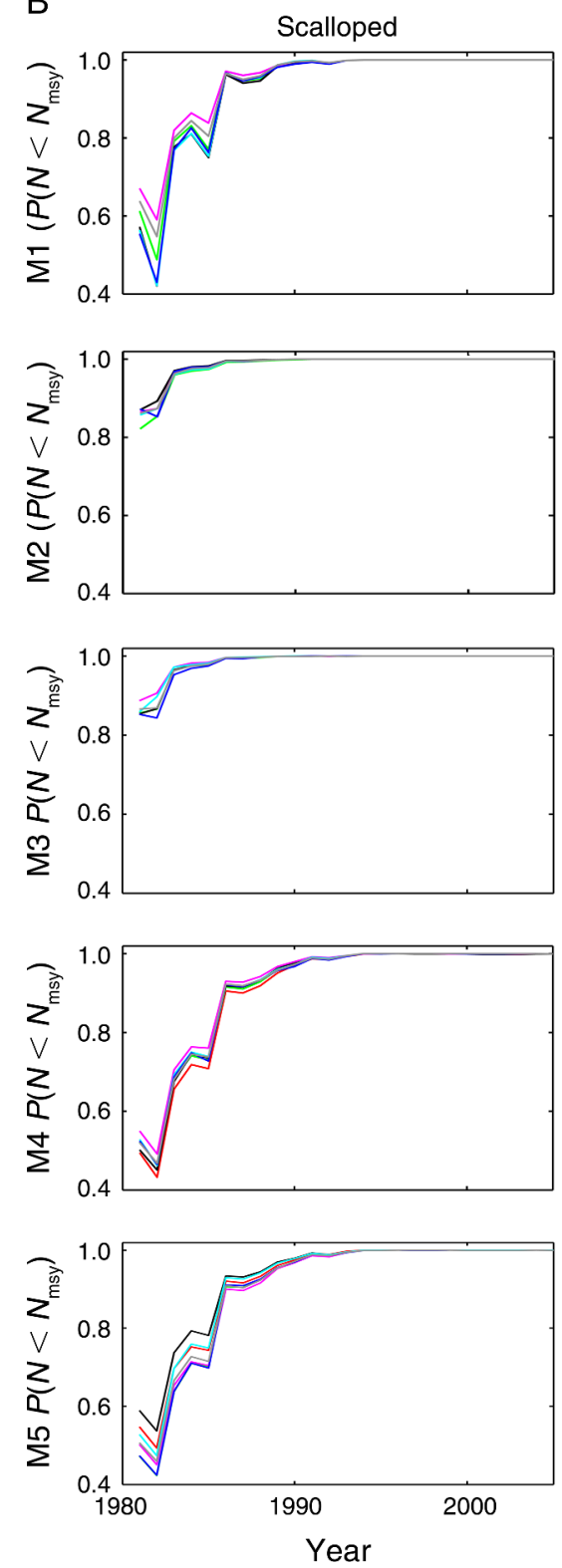
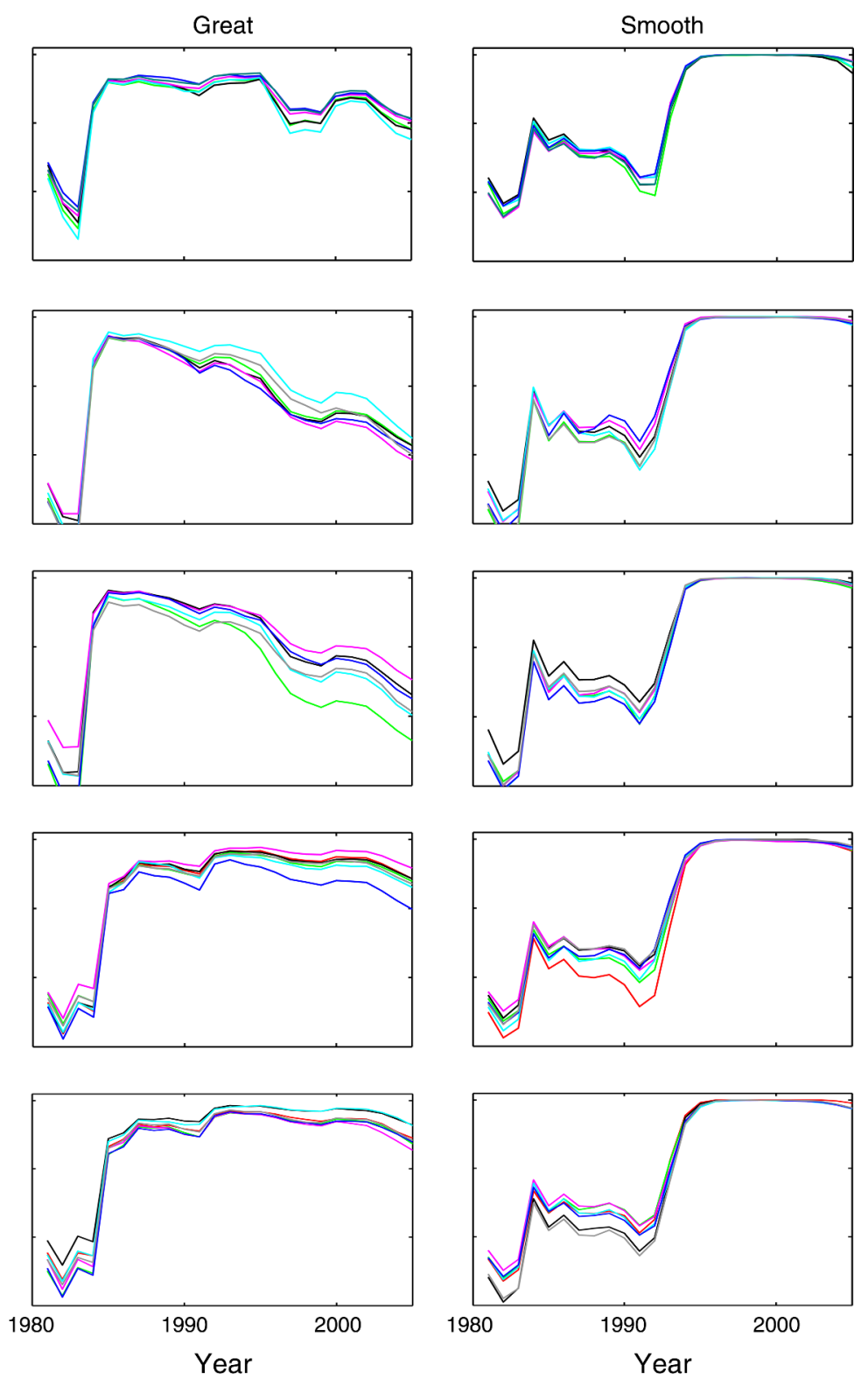

FIG. 6. Continued.

autocorrelation of the Markov chains, whether thinning should be used or not was beyond the scope of our study.

The DIC is widely used to compare hierarchical models (Spiegelhalter et al. 2002, 2004, Jiao et al. 2009 b). However, other criteria, such as the posterior likelihood ratio and posterior predictive $\mathrm{p}$ values have also been proposed for model checking and/or comparison (Meng 1994, Aitken et al. 2005). These other criteria have been found to provide conclusions that are consistent with those derived by frequentist theory. Application of these alternative Bayesian criteria in applied ecological modeling should be further investigated.

The hierarchical population growth model can simulate population growth rate variation caused by the hierarchy of growth rates, which has been related to regime shifts and changes in productivity regimes in some cases (Beamish et al. 1999, Clark 2003). There is evidence that even intraspecific population growth rates, $r_{k}$, can vary over time as a consequence of climate variation, for example (Beamish et al. 1999, Peterman et al. 2003, Jiao et al. 2008). Although variation of the intraspecific population growth rate was not the main reason for using hierarchical models in our case, the 


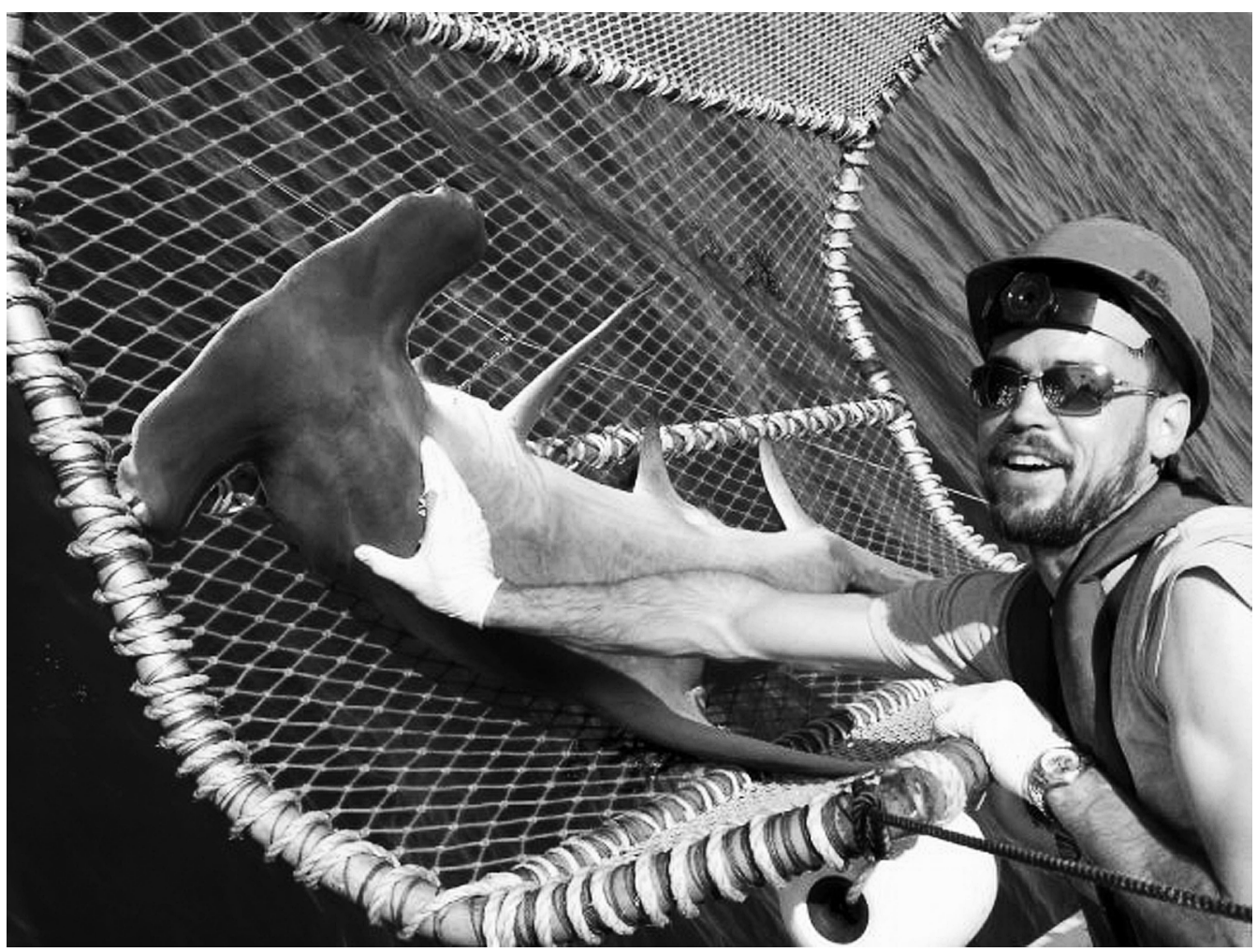

Plate 1. Chris Hayes holds a great hammerhead shark as it is tagged in the National Marine Fisheries Service, Pascagoula Lab shark bottom longline research cruise. Photo credit: Dana Bethea.

stability and more reasonable biological results for the scalloped hammerhead shark population may be a reflection of using hierarchical models.

Traditionally used likelihood or frequentist methods often require prior knowledge on the process and/or observation errors or the ratio of process to observation error. However, in this study we used the Bayesian approach, which does not necessarily need prior knowledge on the variances (Millar and Meyer 2000, Calder et al. 2003, Carroll et al. 2006). The Bayesian approach has been found to be very efficient in identifying the variances in state-space models. Simulations would further help identify the ability of this approach to accurately estimate process error and observation error. A recent simulation study (He 2010) found that statespace models with both process and observation error considered provided the best estimates (in terms of accuracy and precision) of the key parameters of the models and the population sizes.

We emphasize that the purpose of our study was to present a new approach for assessing data-poor and poor-data stocks. The results of the hammerhead shark stock assessment that we present as an example should thus be viewed as illustrative rather than as conclusive evidence of their present status. To that end, a more systematic study investigating a variety of data and analytical issues should be conducted.
Hierarchical Bayesian models allow poor-data species to borrow strength from species with good-quality data and have been applied to small-area, small-sample size estimation problems (Berger 1985, He and Sun 1998, 2000, Su et al. 2001, Gelman et al. 2004). Many hierarchical modeling analyses based on empirical data showed the advantages of using multilevel analysis because it allows years and/or locations with poor data to borrow strength from years and/or locations with better data (He and Sun 2000, Su et al. 2001, Gelman et al. 2004). It is thus important for researchers in the field of fisheries to become more acquainted with the application of Bayesian hierarchical models for datapoor and poor-data species.

\section{ACKNOWLEDGMENTS}

The project was supported in part by the USDA Cooperative State Research, Education and Extension Service, Hatch project \#0210510, and a grant from MARFIN, NOAA. We thank J. Carlson, W. Ingram, and C. McCandless for making hammerhead shark fisheries data accessible. Special thanks to Milani Chaloupka and one anonymous reviewer, and to Inyoung Kim in the Department of Statistics at Virginia Tech, who provided valuable advice on the revision of this paper.

\section{Literature Cited}

Aitken, M., R. Boys, and T. Chadwick. 2005. Bayesian point null hypothesis testing via the posterior likelihood ratio. Statistical Computing 15:217-230. 
Beamish, R. J., D. J. Noakes, G. A. McFarlane, L. Klyashtorin, V. V. Ivanov, and V. Kurashov. 1999. The regime concept and natural trends in the production of Pacific salmon. Canadian Journal of Fisheries and Aquatic Sciences 56:516-526.

Beerkircher, L. R., C. J. Brown, and D. W. Lee. 2002. SEFSC pelagic observer program data summary for 1992-2000. NOAA Technical Memorandum NMFS-SEFSC-486. NOAA, Miami, Florida, USA.

Berger, J. O. 1985. Statistical decision theory and Bayesian analysis. Springer-Verlag, New York, New York, USA.

Bowman, A. W., and A. Azzalini. 1997. Applied smoothing techniques for data analysis. Oxford University Press, Oxford, UK.

Cailliet, G. M., H. F. Mollet, G. G. Pittenger, D. Bedford, and L. J. Natanson. 1992. Growth and demography of the Pacific angel shark (Squatina californica), based upon tag returns off California. Australian Journal of Marine and Freshwater Research 43:1313-1330.

Calder, C., M. Lavine, P. Muller, and J. S. Clark. 2003. Incorporating multiple sources of stochasticity into dynamic population models. Ecology 84:1395-1402.

Carlson, J. K., I. E. Baremore, and D. M. Bethea. 2005. The directed shark gillnet fishery catch and bycatch, 2004. Sustainable Fisheries Division Contribution PCB-05-01. Sustainable Fisheries Division, Panama, City, Florida, USA.

Carlson, J. K., and D. M. Bethea. 2005. Standardized catch rates of large coastal sharks from a fishery-independent survey in northeast Florida. Southeast Data, Assessment and Review (SEDAR) 11, Large Coastal Shark Data Workshop LCS05/06-DW-12. Sustainable Fisheries Division, Panama City, Florida, USA.

Carroll, R. J., D. Ruppert, L. A. Stefanski, and C. M. Crainiceanu. 2006. Measurement error in nonlinear models: a modern perspective. Chapman and Hall/CRC, London, UK.

Clark, J. S. 2003. Uncertainty and variability in demography and population growth: a hierarchical approach. Ecology 84:1370-1381.

Cortés, E. 2002a. Incorporating uncertainty into demographic modeling: application to shark populations and their conservation. Conservation Biology 16:1048-1062.

Cortés, E. 2002b. Stock assessment of small coastal sharks in the U.S. Atlantic and Gulf of Mexico. Sustainable Fisheries Division Contribution SFD-01/02-152 133. Sustainable Fisheries Division, Panama City, Florida, USA.

Cortés, E., A. Morgan, and G. Burgess. 2005. Standardized catch rates of large coastal sharks from the commercial shark fishery observer program, 1994-2004. Southeast Data, Assessment and Review (SEDAR) 11, Large Coastal Shark Data Workshop LCS05/06-DW-17. Sustainable Fisheries Division, Panama City, Florida, USA.

Cortés, E., and J. A. Neer. 2005. Updated catches of Atlantic sharks. Southeast Data, Assessment and Review (SEDAR) 11, Large Coastal Shark Data Workshop LCS05/06-DW-16. Sustainable Fisheries Division, Panama City, Florida, USA.

de Valpine, P., and A. Hastings. 2002. Fitting population models incorporating process noise and observation error. Ecological Monographs 72:57-76.

Fujiwara, M., and H. Caswell. 2001. Demography of the endangered North Atlantic right whale. Nature 414:537-541.

Gelman, A., J. B. Carlin, H. S. Stern, and D. B. Rubin. 2004. Bayesian data analysis. Chapman and Hall, Boca Raton, Florida, USA.

Gelman, A., and D. B. Rubin. 1992. Inference from iterative simulation using multiple sequences (with discussion). Statistical Science 7:457-511.

Gilks, W. R. 1996. Full conditional distributions. Pages 75-78 in W. R. Gilks, S. Richardson, and D. J. Spiegelhalter, editors. Markov chain Monte Carlo in practice. Chapman and Hall, London, UK.
Hayes, C. 2008. Investigating single and multiple species fisheries management: stock status evaluation of hammerhead (Sphyrna spp.) sharks in the western North Atlantic and Gulf of Mexico. Thesis. Virginia Tech University, Blacksburg, Virginia, USA.

$\mathrm{He}$, Q. 2010. Investigating the performance of processobservation-error-estimator and robust estimators in surplus production model: a simulation study. Thesis. Virginia Tech University, Blacksburg, Virginia, USA.

He, Z., and D. Sun. 1998. Hierarchical Bayes estimation of hunting success rates. Environmental and Ecological Statistics 5:223-236.

He, Z., and D. Sun. 2000. Hierarchical Bayes estimation of hunting success rates. Biometrics 5:223-236.

Hilborn, R., and C. Walters. 1992. Quantitative fisheries stock assessment: choice, dynamics, and uncertainty. Chapman and Hall, New York, New York, USA.

Hoenig, J. M., and S. H. Gruber. 1990. Life history patterns in the elasmobranchs: implications for fisheries management. Pages 1-16 in H. L. Pratt, Jr., S. H. Gruber, and T. Taniuchi, editors. Elasmobranchs as living resources: advances in the biology, ecology, systematics, and the status of the fisheries. NOAA Technical Report NMFS 90. NOAA, Silver Spring, Maryland, USA.

Ingram, W., T. Henwood, M. Grace, L. Jones, W. Driggers, and K. Mitchell. 2005. Catch rates, distribution, and size composition of large coastal sharks collected during NOAA Fisheries bottom longline surveys from the U.S. Gulf of Mexico and U. S. Atlantic Ocean. Southeast Data, Assessment and Review (SEDAR) 11, Large Coastal Shark Data Workshop LCS05/06-DW-27. Sustainable Fisheries Division, Panama City, Florida, USA.

IUCN (International Union for the Conservation of Nature). 2006. 2006 IUCN red list of threatened species. (www. iucnredlist.org $\rangle$

Jiao, Y., C. Hayes, and E. Cortés. 2009a. Bayesian hierarchical models for fish-complex stock assessment without species-specific data. ICES Journal of Marine Science 66:367-377.

Jiao, Y., R. Neves, and J. Jones. 2008. Models and model selection uncertainty in estimating growth rates of endangered freshwater mussel populations. Canadian Journal of Fisheries and Aquatic Sciences 65:2389-2398.

Jiao, Y., K. Reid, and E. Smith. 2009b. Model selection uncertainty and Bayesian model averaging in fisheries recruitment modeling. Pages 505-524 in R. J. Beamish and B. J. Rothschild, editors. The future of fisheries science in North America. Springer, New York, New York, USA.

Kieth, L. B., and L. A. Windberg. 1978. A demographic analysis of the snowshoe hare cycle. Wildlife Monographs 58:1-70.

Krebs, C. J., R. Boonstra, S. Boutin, and A. R. E. Sinclair. 2001. What drives the 10 year cycle of snowshoe hare cycle? BioScience 51:25-35.

Liu, Y., N. Liu, and H. Zhao. 2005. Inferring protein-protein interactions through high-throughput interaction data from diverse organisms. Bioinformatics 21:3279-3285.

MacCall, A. D. 2009. Depletion-corrected average catch: a simple formula for estimating sustainable yields in datapoor situations. ICES Journal of Marine Science 66:22672271.

Mace, P. M., and M. P. Sissenwine. 1993. How much spawning per recruit is enough? In Risk evaluation and biological reference points for fisheries management. Ed. by S. J. Smith, J. J. Hunt, and D. Rivard, editors. Canadian Special Publications Fisheries and Aquatic Sciences 120:101-118.

MacEachern, S. N., and L. M. Berliner. 1994. Subsampling the Gibbs sampler. American Statistician 48:188-189.

MathWorks. 2010. Matlab user's manual. The Mathworks, Inc., Natick, Massachusetts, USA. 
May, R. M., J. R. Beddington, C. W. Clark, S. J. Holt, and R. M. Laws. 1979. Management of multispecies fisheries. Science 205:267-277.

McAllister, M. K., and G. P. Kirkwood. 1998. Using Bayesian decision analysis to help achieve a precautionary approach to managing newly developing fisheries. Canadian Journal of Fisheries and Aquatic Sciences 55:2642-2661.

Meng, X. 1994. Posterior predictive p-values. Annuals of Statistics 22:1142-1160.

Millar, R. B., and R. Meyer. 2000. Non-linear state-space modeling of fisheries biomass dynamics by using MetropolisHastings within-Gibbs sampling. Applied Statistics 49:27342.

Mollet, H. F., and G. M. Cailliet. 2002. Comparative population demography of elasmobranchs using life history tables, Leslie matrices and stage-based matrix models. Marine and Freshwater Research 53:503-516.

National Marine Fisheries Service (NMFS). 2006. SEDAR 11: stock assessment report - large coastal shark complex, blacktip and sandbar shark. National Marine Fisheries Service, Silver Spring, Maryland, USA.

National Marine Fisheries Service (NMFS). 2009. Report of the western Pacific Stock assessment review 1: Hawaii deep slope bottomfish. National Marine Fisheries Service, Silver Spring, Maryland, USA.

National Research Council (NRC). 2010. Assessment of seaturtle status and trends: integrating demography and abundance. National Academies Press, Washington, D.C., USA.

Northeast Data Poor Stocks Working Group. 2009. The Northeast Data Poor Stocks Working Group Report, December 8-12, 2008 Meeting. Part A. Skate species complex, deep sea red crab, Atlantic wolffish, scup, and black sea bass. Reference Document 09-02. U.S. Department of Commerce, Northeast Fishery Science Center, Woods Hole, Massachusetts, USA.

Northeast Fisheries Science Center (NEFSC). 2008a. Assessment of 19 northeast groundfish stocks through 2007: report of the 3rd Groundfish Assessment Review Meeting (GARM III), Northeast Fisheries Science Center, Woods Hole, Massachusetts, August 4-8, 2008. Northeast Fisheries Science Center Reference Document 08-15. U.S. Department of Commerce, NOAA Fisheries, Northeast Fisheries Science Center, Woods Hole, Massachusetts, USA.

Northeast Fisheries Science Center (NEFSC). 2008b. 47th Northeast Regional Stock Assessment Workshop (47th
SAW) Assessment Report. Northeast Fisheries Science Center Reference Document 08-12a. U.S. Department of Commerce, NOAA Fisheries, Northeast Fisheries Science Center, Woods Hole, Massachusetts, USA.

Osborne, J. W. 2000. Advantages of hierarchical linear modeling. Practical Assessment, Research, and Evaluation $7: 1-3$.

Peterman, R. M., B. J. Pyper, and B. W. MacGregor. 2003. Use of the Kalman filter to reconstruct historical trends in productivity of Bristol Bay sockeye salmon (Oncorhynchus nerka). Canadian Journal of Fisheries and Aquatic Sciences 60:809-824.

Polovina, J. L., and S. Ralston. 1987. Tropical snappers and groupers: biology and fisheries management. Westview Press, Boulder, Colorado, USA.

Roberts, G. O., and J. S. Rosenthal. 2001. Infinite hierarchies and prior distributions. Bernoulli 7:453-471.

Schwartz, F. J., C. T. McCandless, and J. J. Hoey. 2007. Trends in relative abundance for shark species caught during a UNC longline survey conducted between 1972 and 2005 in Onslow Bay, NC. SEDAR 13-DW-34-V2. Sustainable Fisheries Division, Panama City, Florida, USA.

Sminkey, T. R., and J. A. Musick. 1995. Age and growth of the sandbar shark, Carcharhinus plumbeus, before and after population depletion. Copeia 1995:871-883.

Smith, S. E., D. W. Au, and C. Show. 1998. Intrinsic rebound potentials of 26 species of Pacific sharks. Marine and Freshwater Research 49:663-678.

Spiegelhalter, D. J., N. G. Best, B. P. Carlin, and A. van der Linde. 2002. Bayesian measures of model complexity and fit (with discussion). Journal of the Royal Statistical Society Series B 64:583-640.

Spiegelhalter, D. J., A. Thomas, N. Bestand, and D. Lunn. 2004. WinBUGS user manual (version 1.4.1). MRC Biostatistics Unit, Cambridge, UK.

Su, Z., M. D. Adkison, and B. W. Van Alen. 2001. A hierarchical Bayesian model for estimating historical salmon escapement and escapement timing. Canadian Journal of Fisheries and Aquatic Sciences 58:1648-1662.

Walters. C. J. 1998. Evaluation of quota management policies for developing fisheries. Canadian Journal of Fisheries and Aquatic Sciences 55:2691-2705.

Wikle, C. K. 2003. Hierarchical Bayesian models for predicting the spread of ecological processes. Ecology 84:1382-1394.

\section{SUPPLEMENT}

WinBUGS codes for the models used in the paper (Ecological Archives A021-120-S1). 